\title{
Compactness and the Maximal Gibbs State for Random Gibbs Fields on a Lattice
}

\author{
Jean Bellissard ${ }^{1}$ and Raphael Hфegh-Krohn ${ }^{2}$ \\ 1 Université de Provence, and Centre de Physique Théorique, CNRS, Luminy, Case 907, F-13288 \\ Marseille, Cedex 2, France \\ 2 Institute of Mathematics, University of Oslo, Oslo, Norway
}

\begin{abstract}
We prove for a general class of Gibbsian Random Field on $\mathbb{Z}^{v}$ that the set of tempered Gibbs states is compact. This class contains the Euclidean random fields. Moreover if the interaction is attractive, there is a unique minimal and maximal Gibbs state $\mu_{-}$and $\mu_{+} \cdot \mu_{ \pm}$are unique translation invariant and have the global Markov property. We also prove that uniqueness of the tempered Gibbs state is equivalent to the magnetizations $m_{ \pm}=\mu_{ \pm}\left(q_{x}\right)$ being equal which is true if the pressure is differentiable.
\end{abstract}

\section{Introduction}

It is well known in statistical mechanics, that any statistical mechanical system with a compact state space has a compact set of Gibbs states. We prove in this paper, by utilizing a criterion that goes back to Dobrushin [11], that also for statistical mechanical systems with a non-compact state space the set of Gibbs states is compact provided the interaction satisfies certain conditions, and we consider as Gibbs states only the tempered Gibbs states. In fact we prove that the set of tempered Gibbs states form a Choquet simplex. This holds especially for the Euclidean lattice fields, and also for a much wider class of lattice interactions given by one and two-body forces.

The compactness of the Gibbs states gives us the existence of the maximal $\mu_{+}$ and minimal $\mu_{-}$Gibbs state in the case of an attractive interaction. Let us point out that the Euclidean lattice fields have attractive interaction. In the case of compact state space and attractive interactions $\mu_{+}$and $\mu_{-}$were introduced by Preston [40] which also proved that they were pure. Later Fölmer [19] pointed out that they also have the global Markov property if the corresponding interaction is Markov. Using the compactness for the tempered Gibbs states in the case of non-compact fiber, we are able to prove not only that the maximal and minimal Gibbs states exists, but also that they are pure, translation invariant and have the global Markov property. Moreover the set of tempered Gibbs 
states reduces to one point if and only if $\mu_{+}=\mu_{-}$, i.e. uniqueness is equivalent to $\mu_{+}=\mu_{-}$. Since for any translation invariant interaction $\mu_{+}$and $\mu_{-}$are translation invariant, we have that the magnetizations $m_{+}=\mu_{+}\left(q_{x}\right)$ do not depend on the position $x$ and of course $m_{-} \leqq m_{+}$. Using the technique of the Wasershtein distance we also prove that $m_{-}=m_{+}$implies uniqueness of the tempered Gibbs states. We also prove the existence of the pressure and the result that differentiability of the pressure implies the uniqueness of the Gibbs state.

Among the main motives for writing this paper was the quest for pure Gibbs state with the global Markov properties. Fölmer and Preston had pointed out in the case of compact state space the unique advantages of the maximal and minimal Gibbs states. However, in the Euclidean lattice field theories the state space is not compact, moreover the uniqueness and global Markov properties are especially important in these models. Of course in the case of the continuous Euclidean quantum fields the question of uniqueness and global Markov property is even more important. It turns out that many of the techniques developed in this paper extend to the continuous case. This is the topic of a forthcoming paper by the same two authors.

It is only fair to mention here that much of the inspiration for extending the known results from the compact state space to the non-compact state space was provided by non-standard analysis. For a treatment of these problems by nonstandard analysis we refer the interested reader to [52].

\section{Definitions and Notations}

Let $R$ be a denumerable subset of $\mathbb{Z}^{v}$. For $x, y$, belonging to $R$ we denote by $d(x, y)$ their distance in $\mathbb{Z}^{v}$

$$
d(x, y)=\sum_{\alpha=1}^{v}\left|x_{\alpha}-y_{\alpha}\right| .
$$

A slowly increasing sequence $\mathbf{q}=\left(q_{x}\right)_{x \in R}$ is an element of $\mathbb{R}^{R}$ such that

$$
\exists N>0, \sup _{x \in R} d(0, x)^{-N}\left|q_{x}\right|<+\infty .
$$

$\mathscr{S}_{R}^{\prime}$ will denote the set of such sequences. In much the same way $\mathscr{S}_{R}$ will denote the set of fastly decreasing sequences.

Giving a matrix $\mathbf{r}=(r(x, y))_{(x, y) \in R^{2}}$, we shall put

$$
\begin{gathered}
\|\mathbf{r}\|=\sup _{x \in R} \sum_{y \in R}|r(x, y)|, \\
\|\mathbf{r}\|_{\eta, N}=\sup _{x \in R} \sum_{y \in R}|r(x, y)|[1+\eta d(x, y)]^{N} .
\end{gathered}
$$

I.3.a).b). define algebraic norms on the algebra $\mathscr{A}_{R}$ of fastly decreasing matrices:

$$
\mathbf{r} \in \mathscr{A}_{R} \Leftrightarrow \sup _{x \in R} \sum_{y \in R}|r(x, y)| d(x, y)^{N}<+\infty \forall N \in \mathbb{N} .
$$

At each site $x \in R$ we define a random variable $\sigma_{x}$ with values in $\mathbb{R}$. The configura- 
tion space for the process $\sigma_{R}=\left(\sigma_{x}\right)_{x \in R}$ can be taken as the product $\Omega_{R}=\mathbb{R}^{R}$ with the product Borel structure. $\Omega_{R}$ is a polish space for the product topology since $R$ is denumerable.

Let $\mathfrak{F}(R)$ be the set of finite subsets of $R$ and $\mathfrak{F}(R)$ the set of every subset of $R$. For $\Lambda \in \mathfrak{F}(R)$, let $\mathscr{B}_{\Lambda}$ be the $\sigma$-algebra generated by the subprocess $\sigma_{A}=\left(\sigma_{x}\right)_{x \in \Lambda}$. If we identify $\Omega_{R}$ with $\Omega_{\Lambda} \times \Omega_{\Lambda^{c}}$ (where $\Lambda^{c}=R \backslash \Lambda$ ), a $\mathscr{B}_{\Lambda^{\prime}}$-measurable function is nothing but a function depending only on $\Omega_{\Lambda}$. At last, $\mathfrak{M}\left(\Omega_{R}\right)$ will denote the set of probability measures on $\Omega_{R}$.

In order to describe the thermodynamical behaviour of a system, we use the Gibb ansatz:

for each finite region $\Lambda \in \mathfrak{F}(R)$ and each configuration $\mathbf{q}_{\Lambda^{c}}$ in $\Omega_{\Lambda^{c}}$, the probability distribution of $\sigma_{\Lambda}$ conditionned by $\sigma_{\Lambda^{c}}=\mathbf{q}_{\Lambda^{c}}$ is given by

$$
E_{\Lambda^{c}}^{\mathbf{q}}\left(d \mathbf{q}_{\Lambda}\right)=Z_{\Lambda}(\mathbf{q})^{-1} \prod_{x \in \Lambda}\left(d q_{x} \exp \left(-V\left(q_{x}\right)\right)\right) \prod_{x \in \Lambda} \prod_{y \in R} \exp \left(-W_{x, y}\left(q_{x}-q_{y}\right)\right),
$$

where $Z_{\Lambda}(\mathbf{q})$ is a normalisation factor.

In order to be precise we shall investigate the following special examples:

A) The $W$ 's are a $\mathscr{C}^{1}$ positive convex function with strictly increasing, unbounded, absolutely continuous first derivative. Moreover, there are $\mathbf{J} \in \mathscr{A}_{R}$ and $n>1$

A1) $W_{x y}(q) \leqq J(x, y)(1+|q|)^{n}$

or

A2) $W_{x y}^{(2)}(q) \leqq J(x, y)$ for almost all $q$.

B) $V$ is a $\mathscr{C}^{2}$ positive function and there is $M_{0}>0, \alpha>\operatorname{Max}(n-2,0)$ (or $\alpha \geqq 0$ if A2 holds), $A>0$ such that

$$
|q|>M_{0} \Rightarrow V^{(2)}(q)>A|q|^{\alpha} .
$$

Note that $\mathrm{A} 2) \Rightarrow \mathrm{A} 1$ ).

From A1) it follows immediately that I.5a is defined provided $\mathbf{q} \in \mathscr{S}^{\prime}$, and is continuous with respect to $\mathbf{q}$. We shall put

$$
E_{\Lambda^{c}}^{\mathbf{q}}\left(d \mathbf{q}_{\Lambda}\right)=0 \quad \text { if } \quad \mathbf{q} \notin \mathscr{S}_{R}^{\prime} .
$$

Since $\mathscr{S}_{R}^{\prime}$ is a Borel subset of $\Omega$, it follows that I5a-I.5b) defines a Borel function of $\mathbf{q} \in \Omega$.

Examples: i) The free field with mass $m: R=\mathbb{Z}^{v}$.

$$
V(q)=\frac{1}{2} m^{2} q^{2} \quad W_{x, y}(q)=\frac{1}{2} q^{2} \quad \text { if } \quad d(x, y)=1
$$

and $W_{x, y}=0$ otherwise.

ii) The $P(\varphi)$ lattice field: as before but

$$
V(q)=\frac{1}{2} m^{2} q^{2}+P(q),
$$

where $P$ is a polynomial bounded from below.

iii) $W(q)=a|q|^{\sigma} 1<\sigma \leqq 2$ satisfies A1.

The main property of the family $\left(E_{\Lambda^{c}}\right)_{\Lambda \in \mathscr{F}(R)}=\mathscr{E}$ is that it is a local specification in the following sense $[18,40,41]$. 
Definition I.1. Let $f_{+}(\Omega)$ be the set of positive, possibly infinite, Borel function on $\Omega$.

A local specification is a family $\mathscr{E}=\left(E_{\Lambda^{c}}\right)_{\Lambda \in \mathscr{F}(R)}$ where for each $\Lambda, E_{\Lambda^{c}}$ maps $f_{+}(\Omega)$ into itself and satisfies:

S1) Normalisation: $E_{A^{c}}(1)=1$.

S2) $\sigma$-additivity: If $\left(F_{n}\right)_{n \geqq 0}$ is a sequence in $f_{+}(\Omega)$, then

$$
\sum_{n \geqq 0} E_{\Lambda^{c}}\left(F_{n}\right)=E_{\Lambda^{c}}\left(\sum_{n \geqq 0} F_{n}\right)
$$

S3) Locality: If $F \in f_{+}(\Omega), E_{A^{c}}(F)$ is $\mathscr{B}_{\Lambda^{c}}$-measurable, and if $F$ is $\mathscr{B}_{\Lambda^{c}}$-measurable $E_{A^{c}}(F)=F$.

S4) Compatibility: If $\Lambda_{1} \subset \Lambda_{2}$ then

$$
E_{\Lambda_{1}^{c}} E_{\Lambda_{2}^{c}}=E_{\Lambda_{2}^{c}} E_{\Lambda_{1}^{c}}=E_{\Lambda_{2}^{c}} \text {. }
$$

The central problem in statistical mechanics is to find the global properties of the process $\sigma_{R}$ from the knowledge of the local specification $\mathscr{E}$.

In particular the distribution of $\sigma_{R}$ can be represented by a probability measure $\mu$ on $\Omega$, whose conditional probabilities $E_{\mu}\left(\cdot \mid \mathscr{B}_{\Lambda^{c}}\right)$ coincide $\mu$-almost surely with $E_{\Lambda^{c}}$.

Definition I.2. Let $\mathscr{E}$ be a local specification. The set $\mathscr{G}(\mathscr{E})$ of Gibbs states of $\mathscr{E}$ is the set of probability measures $\mu$ on $\Omega_{R}$ such that for any $\Lambda \in \mathfrak{F}(R)$

$$
E_{\mu}\left(\cdot \mid \mathscr{B}_{A^{c}}\right)=E_{A^{c}}(\cdot) \quad \mu \text {-almost surely. }
$$

As it is well-known, $\mathscr{G}(\mathscr{E})$ can contain more than one point [11]; this phenomena is connected with the notion of phase transition. However, in the case of unbounded spin we are investigating, many Gibbs states have no physical relevance.

For instance, for the free field on a lattice, Guerra, Rosen and Simon [27] have shown an example of such an irrelevant Gibbs state.

One way to avoid such examples is to constrain $\sigma_{R}$ to a growth condition at infinity, as suggested by euclidean field theories:

Definition I.3. A tempered measure is a probability measure $\mu$ on $\Omega_{R}$ such that $\left(\mu\left(\left|\sigma_{x}\right|\right)_{x \in R}\right.$ is in $\mathscr{S}_{R}^{\prime}$. The set of tempered measures will be denoted by $\mathfrak{M}_{t}\left(\Omega_{R}\right)$. The set $\mathfrak{M}_{t, \text { exp }}\left(\Omega_{R}\right)$ will denote the set of probability measures on $\Omega_{R}$ such that $\left(\mu\left(\exp \left(a\left|\sigma_{x}\right|\right)\right)\right)_{x \in R} \in \mathscr{S}_{R}^{\prime}$.

In the Appendix, we study the space $\mathfrak{M}_{t}$ and give a characterization of compact sets of $\mathfrak{M}_{t}$. Moreover we prove that $\mathscr{S}_{R}^{\prime}$ has $\mu$-measure one for any $\mu \in \mathfrak{M}_{t}$, and $\mathrm{I} .5 \mathrm{~b}$ is irrelevant.

It is easy to verify that $\mu \in \mathscr{G}(\mathscr{E})$ if and only if $\mu E_{\Lambda^{c}}=\mu$ for any $\Lambda \in \mathfrak{F}(R)$. On the other hand if $E_{A^{c}}\left(\left|\sigma_{x}\right|\right)<+\infty$, and $\mu \in \mathfrak{M}_{t}$ then $\mu E_{A^{c}} \in \mathfrak{M}_{t}$.

The first problem is to prove that $\mathscr{G}_{t}(\mathscr{E})=\mathfrak{M}_{t} \cap \mathscr{G}(\mathscr{E})$ is not empty. Actually we shall prove that it is a compact set with the help of a criteria first established by Dobrushin [14].

Then we will use the technics of the FKG order [20] as developed by Preston $[40]$ to prove the existence of states with "+" or " -" boundary condition. If 
$\mathscr{E}$ is translation covariant, locally Markov and reflection positive, then $\mu_{ \pm}$are translation invariant, globally Markov and reflection positive. Moreover $\mathscr{G}_{t}(\mathscr{E})$ is reduced to one point if and only if $\mu_{+}=\mu_{-}$. The differentiability of the pressure with respect to the external magnetic field is sufficient to insure this condition.

\section{Existence and Compactness}

We first remark that any limit point of the family $\left(\mu E_{\Lambda^{c}}\right)_{\Lambda \in \mathscr{F}(R)}$ where $\mu \in \mathfrak{M}_{t}$ is a tempered Gibbs state. Conversely if $\mu \in \mathscr{G}_{t}(\mathscr{E})$ then $\mu=\mu E_{\Lambda^{c}} \forall \Lambda \in \mathfrak{F}(R)$ and it is such a limit point. We get a way of describing $\mathscr{G}_{t}(\mathscr{E})$. In the examples I.5 if the hypothesis $\mathrm{A} 1-\mathrm{A} 2$ holds, we shall prove that $\mathscr{G}_{t}(\mathscr{E})$ is moreover compact. This justifies the following definition. (See Appendix for the definition of $\mathscr{W} . \mathscr{T}$. Topologies)

Definition II.1. A local specification $\mathscr{E}$ is compact (respectively $\mathscr{T}$ compact) if it satisfies the following conditions:

(i) $\forall \mu \in \mathfrak{M}_{t},\left(\mu E_{\Lambda^{c}}\right)_{\Lambda \in \mathfrak{F}(R)}$ is a compact family in $\left(\mathfrak{M}_{t}, \mathscr{W}\right)$ (respectively $\left.\left(\mathfrak{M}_{t}, \mathscr{T}\right)\right)$.

(ii) The set $\mathscr{G}_{t}(\mathscr{E})$ is $\mathscr{W}$-compact (respectively $\mathscr{T}$-compact). It follows from this definition that $\mathscr{G}_{t}(\mathscr{E})$ is not empty if $\mathscr{E}$ is compact.

Proposition II.2 [44]. If $\mathscr{E}$ is a compact local specification then $\mathscr{G}_{t}(\mathscr{E})$ is a Choquet simplex.

Proof. We recall that a Choquet simplex is a metrizable compact convex set such that any element can be decomposed in a unique way into an integral over the set of its extreme points [9].

Since $\mathfrak{M}_{t}$ is a polish space, the $\mathscr{W}$-compactness of $\mathscr{E}$ implies that $\mathscr{G}_{t}(\mathscr{E})$ is compact and metrizable. Moreover $\mu \in \mathscr{G}_{t}(\mathscr{E})$ if and only if

$$
\mu E_{\Lambda^{c}}=\mu \quad \forall \Lambda \in \mathfrak{F}(R) .
$$

From this it follows that $\mathscr{G}_{t}(\mathscr{E})$ is convex.

Now let $\mathscr{B}_{\infty}=\bigcap_{A \in \mathscr{F}(R)} \mathscr{B}_{\Lambda^{c}}$ be the $\sigma$-algebra at infinity, and $\mu_{1}, \mu_{2}$ be two tempered Gibbs states. Then, if $\mu=\frac{1}{2}\left(\mu_{1}+\mu_{2}\right)$ we get

$$
\mu_{i}=F_{i} \mu \quad F_{i} \in L_{+}^{1}\left(\Omega_{R}, \mathscr{B}_{R}, \mu\right) .
$$

Since $\mu_{i}$ satisfies II.1, $\mu$ is again a Gibbs state, and $F_{i}$ is $\mathscr{B}_{\infty}$-measurable. Then both $F_{1} \wedge F_{2}$ and $F_{1} \vee F_{2}$ are $\mathscr{B}_{\infty}$-measurable, and therefore $\mu_{1} \wedge \mu_{2}, \mu_{1} \vee \mu_{2}$ are tempered bounded positive measures satisfying II.1. This is precisely the definition of a Choquet Simplex [9a),9b)]. We recall that $\mu \in \mathscr{G}_{t}(\mathscr{E})$ is extremal if and only if every $\mathscr{B}_{\infty}$-measurable set has measure zero or one [40].

The interest of the Definition II.1 comes from the following criteria first described by Dobrushin $[7,14]$.

Theorem II.3. Let $\mathscr{E}$ be a local specification. The following is sufficient for $\mathscr{E}$ to be $\mathscr{W}$-compact.

$$
E_{\{x\}^{c}}\left(\left|\sigma_{x}\right|\right) \leqq A_{x}+\sum_{y \in R} r(x, y)\left|\sigma_{y}\right|
$$


where $\mathbf{A}=\left(A_{x}\right)_{x \in R} \in \mathscr{S}_{R}^{\prime}$, and $r(x, y) \geqq 0$,

$$
\|\mathbf{r}\|<1\|\mathbf{r}\|_{\eta, N}<\infty \forall N, \eta>0
$$

Remark.

1) The matrix $\mathbf{r}$ describes the sensitive dependence of $E_{\{x\}^{c}}$ with respect to the boundary conditions.

2) The proof of this theorem does not depend on the real character of $\sigma_{x}$. It can take values in any complete metric space $(X, \rho)$, and $\left|\sigma_{x}\right|$ has to be replaced by $\rho\left(\xi, \sigma_{x}\right)$ where $\xi \in X$, and $\rho$ is the metric. [14]

Proof. From the appendix, a closed subset $K$ in $\mathfrak{M}_{t}$ is $\mathscr{W}$-compact if there is a positive sequence $c_{x} \in \mathscr{S}_{R}^{\prime}$ such that

$$
\mu\left(\left|\sigma_{x}\right|\right) \leqq c_{x} x \in R, \mu \in K
$$

First let us prove that $\mathscr{G}_{t}(\mathscr{E})$ is $\mathscr{W}$-compact. If $\mu \in \mathscr{G}_{t}(\mathscr{E})$, we get from II.3

$$
\mu\left(\left|\sigma_{x}\right|\right)=\mu\left(E_{\{x\}^{c}}\left(\left|\sigma_{x}\right|\right)\right) \leqq A_{x}+\sum_{y \in R} r(x, y) \mu\left(\left|\sigma_{y}\right|\right) .
$$

Let $\mathbf{m}$ be the sequence $\mathbf{m}=\left(\mu\left(\left|\sigma_{x}\right|\right)\right)_{x \in R}$, II.6 can be written as:

$$
(\mathbb{1}-\mathbf{r}) \mathbf{m} \leqq \mathbf{A} \quad \mathbf{A}=\left(A_{x}\right)_{x \in \mathbf{R}}
$$

Since $\mathbf{r}$ has positive matrix element, II.7 can be solved by:

$$
\mathbf{m} \leqq(1-\mathbf{r})^{-1} \mathbf{A}
$$

provided the Neuman series in the right hand side converges. But $\mathbf{A}$ is slowly increasing. Let $N$ be such that (for some $K>0$ ).

$$
A_{x} \leqq K(1+d(0, x))^{N} .
$$

Since $\|\mathbf{r}\|<1$ and $\|\mathbf{r}\|_{\eta, N}<+\infty, \eta_{0}$ can be chosen so small that

$$
0<\eta<\eta_{0} \Rightarrow\|\mathbf{r}\|_{\eta, N}<1 .
$$

This is sufficient to insure the convergence of the Neuman series for $(1-\mathbf{r})^{-1}$. Moreover

$$
\begin{aligned}
(1-\mathbf{r})^{-1} \mathbf{A}(x) & \leqq\left[1-\|\mathbf{r}\|_{\eta, N}\right]^{-1} \sup _{y} \frac{A_{y}}{[1+\eta d(x, y)]^{N}} \\
& \leqq \frac{K}{\eta^{N}}\left(1-\|\mathbf{r}\|_{\eta, N}\right)^{-1} \sup _{y}\left[\frac{1+n d(0, y)}{1+\eta d(x, y)}\right]^{N} \leqq K^{\prime}[1+\eta d(0, x)]^{N}
\end{aligned}
$$

for some $K^{\prime}>0$.

Then, $(\mathbb{1}-\mathbf{r})^{-1} \mathbf{A}$ is in $\mathscr{S}_{R}^{\prime}$, which proves the $\mathscr{W}$-compactness of $\mathscr{G}_{t}(\mathscr{E})$.

Let us prove now that for $\mu \in \mathfrak{M}_{t}$, the family $\left(\mu E_{\Lambda^{c}}\right)_{\Lambda \in \mathfrak{F}(R)}$ is compact in $\mathfrak{M}_{t}$. Let $\Lambda$ be a finite subset of $R$ and, $K_{\eta}, N$ such that 
If $x \notin \Lambda \mu\left(E_{\Lambda^{c}}\left(\left|\sigma_{x}\right|\right)\right)=\mu\left(\left|\sigma_{x}\right|\right)$, whereas if $x \in \Lambda$ the estimate II.3 gives:

$$
\begin{aligned}
m_{x}=\mu\left(E_{\Lambda^{c}}\left|\sigma_{x}\right|\right) & \leqq A_{x}+\sum_{y \in \Lambda} r(x, y) m_{y}+K \sum_{y \notin \Lambda} r(x, y)[1+\eta d(0, y)]^{N} \\
& \leqq A_{x}+K\|\mathbf{r}\|_{\eta, N}[1+\eta d(0, x)]^{N}+\mathbf{r}_{\Lambda} \mathbf{m}(x),
\end{aligned}
$$

where $r_{\Lambda}(x, y)=r(x, y)$ if $x \in \Lambda, y \in \Lambda$, and $r_{\Lambda}(x, y)=0$ otherwise. In much the same way, the right hand side of II.13 defines a sequence $\mathbf{A}^{\prime} \in \mathscr{S}_{R}^{\prime}$ such that

$$
(1-\mathbf{r})_{A} \mathbf{m} \leqq \mathbf{A}^{\prime}
$$

which gives $x \in \Lambda$.

$$
\mathbf{m}(x) \leqq\left(\mathbb{1}-\mathbf{r}_{A}\right)^{-1} \mathbf{A}^{\prime}(x) \leqq(\mathbb{1}-\mathbf{r})^{-1} \mathbf{A}^{\prime}(x)
$$

for $r_{\Lambda}^{n}(x, y) \leqq r^{n}(x, y)$ if $x, y \in R, n \geqq 1$. If $x \notin \Lambda$

$$
\mathbf{m}(x)=\mu E_{A^{c}}\left(\left|\sigma_{x}\right|\right)=\mu\left(\left|\sigma_{x}\right|\right) \leqq A_{x}^{\prime} \leqq(\mathbb{1}-\mathbf{r})^{-1} \mathbf{A}^{\prime}(x),
$$

and we have proven the proposition.

Remark. If $A_{x} \leqq A \forall x$ in II.3, then: there is $A^{\prime}>0$ such that

$$
\mu\left(\left|\sigma_{x}\right|\right) \leqq A^{\prime} \forall x \in R, \mu \in \mathscr{G}_{t}(\mathscr{E})
$$

as can be seen easily from this proof.

\section{Compactness : Exponential Bound}

In the examples I.5 we shall prove more than the Dobrushin estimate: every tempered Gibbs state is in $\mathfrak{M}_{t, \exp }$. The following result is the main step in this context:

Proposition III.1 (Exponential Bound). Let $\mathscr{E}$ be a local specification. Let us assume the existence of constants $A>0, B>0, C$ and of a positive matrix $\mathbf{r}=(r(x, y))_{x, y \in R^{2}}$ such that

$$
\left.E_{\{x\}^{\mathrm{c}}}\left(\mathrm{e}^{a\left|\sigma_{x}\right|}\right) \leqq \exp \left[\frac{A}{2} a^{2}+\mathrm{Ba}+\mathrm{a} \sum_{y \in R} r(x, y)\left|\sigma_{y}\right|+C\right]\right]
$$

with

$$
\|\mathbf{r}\|<1 \text { and }\|\mathbf{r}\|_{\eta, N}<+\infty \forall N
$$

Then, $\mathscr{E}$ is $\mathscr{T}$-compact, and every tempered Gibbs state $\mu$ satisfies for some $A^{\prime}>0$, $B^{\prime}>0, C^{\prime} \geqq 0$ :

$$
\mu\left(\mathrm{e}^{a\left|\sigma_{x}\right|}\right) \leqq \exp \left(\frac{A^{\prime}}{2} a^{2}+B^{\prime} a+C^{\prime}\right) \forall x \in R, \mu \in \mathscr{G}_{t}(\mathscr{E}) .
$$

Proof. Clearly III.1 implies II.3, and $\mathscr{E}$ is a $\mathscr{W}$-compact local specification.

Let $\mathbf{q}$ be an element of $\mathscr{S}_{R}^{\prime}$ and let $\mathbf{m}_{A}^{\mathbf{q}}$ be the sequence:

$$
\mathbf{m}_{\Lambda}^{\mathbf{q}}(x)=\log E_{A^{c}}^{\mathbf{q}}\left(\mathrm{e}^{a\left|\sigma_{x}\right|}\right) .
$$


From $S 4$, III. 1 we get:

$$
\mathbf{m}_{\Lambda}^{\mathbf{q}}(x)=\log E_{\Lambda^{c}}^{\mathbf{q}}\left(E_{\{x\}^{c}}\left(\mathrm{e}^{a\left|\sigma_{x}\right|}\right)\right) \leqq \frac{A}{2} a^{2}+B a+\log E_{\Lambda^{c}}^{\mathbf{q}}\left(\exp \left(a \sum_{y} r(x, y)\left|\sigma_{y}\right|\right)\right)+C .
$$

Since $\sum_{y} r(x, y)<1$, we can use the Hölder estimate to find:

$$
\mathbf{m}_{\Lambda}^{\mathbf{q}}(x) \leqq A \frac{a^{2}}{2}+B a+C+\mathbf{r}_{\Lambda} \cdot \mathbf{m}_{\Lambda}^{\mathbf{q}}(x)+a \sum_{z \notin \Lambda} r(x, z)\left|q_{z}\right|,
$$

which implies as in Sect II (using $r(x, y) \geqq 0\|\mathbf{r}\|<1$ )

$$
\begin{aligned}
\mathbf{m}_{\Lambda}^{\mathbf{q}}(x) & \leqq\left(1-\mathbf{r}_{A}\right)^{-1}\left(A \frac{a^{2}}{2}+B a+C\right)+a \sum_{y \in \Lambda} \sum_{z \notin \Lambda}\left(1-\mathbf{r}_{\Lambda}\right)^{-1}(x, y) r(y, z)\left|q_{z}\right| \\
& \leqq A^{\prime} \frac{a^{2}}{2}+B^{\prime} a+C^{\prime}+a \sum_{z \notin \Lambda} \frac{\mathbf{r}}{1-\mathbf{r}}(x, z)\left|q_{z}\right| .
\end{aligned}
$$

Since $\mathbf{q} \in \mathscr{S}_{R}^{\prime}$, the last term in the right hand side goes to zero as $\Lambda$ goes to $R$, which means

$$
\limsup _{A_{\uparrow} R} E_{A^{c}}^{\mathbf{q}}\left(\exp \left(a\left|\sigma_{x}\right|\right)\right) \leqq \exp \left(A^{\prime} \frac{a^{2}}{2}+B^{\prime} a+C^{\prime}\right)
$$

Now let $\mu$ be a tempered Gibbs state. We know that $\mathscr{S}_{R}^{\prime}$ has $\mu$-measure one. Therefore, giving $L>0$, the dominated convergence theorem implies:

$$
\begin{aligned}
\mu(\min (\exp & \left.\left.\left(a\left|\sigma_{x}\right|\right), L\right)\right)=\mu E_{A^{c}}\left(\min \left(\exp \left(a\left|\sigma_{x}\right|\right), L\right)\right) \\
& \leqq \mu\left(\overline{\lim \sup } E_{A^{c}}\left(\min \left(\exp \left(a\left|\sigma_{x}\right|\right), L\right)\right)\right) \\
& \leqq \exp \left(A^{\prime} \frac{a^{2}}{2}+B^{\prime} a+C^{\prime}\right)
\end{aligned}
$$

By Fatou's Lemma it follows that if $L \uparrow+\infty$

$$
\mu\left(\exp \left(a\left|\sigma_{x}\right|\right)\right) \leqq \exp \left(A^{\prime} \frac{a^{2}}{2}+B^{\prime} a+C^{\prime}\right) .
$$

The compactness with respect to $\mathscr{T}$ comes from the remark A 7 in the appendix.

Theorem III.2 Let us consider the local specification $\mathscr{E}$ defined in I.5, where $V$ and $\left(W_{x, y}\right)_{x, y \in R}$ satisfy $A-B$. Then, $\mathscr{E}$ is $\mathscr{T}$-compact and the exponential bound III.1 holds.

Proof. In order to estimate $\exp \left(a\left|\sigma_{x}\right|\right)$ it is sufficient to estimate $\exp \left(a \sigma_{x}\right)$ with $a \in \mathbb{R}$. But the estimate is the same for $a>0$ and $a<0$. Thus let us assume $a>0$.

First of all; $\mathscr{S}_{R}^{\prime} \ni \mathbf{q} \rightarrow E_{\{x\}^{c}}^{\mathbf{q}}\left(\exp \left(a \sigma_{x}\right)\right) \ni \mathbb{R}$ is increasing (the ordering on $\mathscr{S}_{R}^{\prime}$ is the product ordering in $\left.\mathbb{R}^{R}\right)$. Indeed:

$$
\begin{aligned}
\frac{\partial}{\partial q_{y}} E_{\{x\}^{c}}^{\mathbf{q}}\left(\exp \left(a \sigma_{x}\right)\right)= & E_{\{x\}^{c}}^{\mathbf{q}}\left(\exp \left(a \sigma_{x}\right) W_{x, y}^{(1)}\left(\sigma_{x}-q_{y}\right)\right) \\
& -E_{\{\}^{c}}^{\mathbf{q}}\left(\exp \left(a \sigma_{x}\right)\right) E_{\{x\}^{c}}^{\mathbf{q}}\left(W_{x, y}^{(1)}\left(\sigma_{x}-q_{y}\right)\right) .
\end{aligned}
$$


Since $q \in \mathbb{R} \rightarrow \exp (a q)$ and $q \in \mathbb{R} \rightarrow W^{(1)}\left(q-q_{y}\right)$ are increasing functions (by $A$ ), the right hand side is positive (FKG inequality, see sect. IV).

Thus we can choose $\mathbf{q}_{0} \in \mathscr{S}_{R}^{\prime}$ big enough and assume $\mathbf{q} \geqq \mathbf{q}_{0}$ to get an upper bound.

Now let $p_{0}$ be a positive real. We get immediately:

$$
E_{\{x\}^{c}}^{\mathbf{q}}\left(\exp \left(a \sigma_{x}\right)\right) \leqq e^{a p_{0}}+\frac{\left.\int_{p_{0}}^{\infty} d q \exp \left(-V(q)-W_{x}(q ; \mathbf{q})+a q\right)\right)}{\int_{p_{0}}^{\infty} d q \exp \left(-V(q)-W_{x}(q ; \mathbf{q})\right)},
$$

with

$$
W_{x}(q ; \mathbf{q})=\sum_{y \in R} W_{x, y}\left(q-q_{y}\right)
$$

We shall estimate the last term in III.12. We note the following result

Lemma III.3. Under the hypothesis A, we have

i) $\exists K>0 \quad\left|W_{x, y}^{(1)}(q)\right| \leqq K J(x, y)(1+|q|)^{n-1}$.

ii) $q \in \mathbb{R} \rightarrow W_{x}(q ; \mathbf{q})$ is $\mathscr{C}^{1}$ for every $\mathbf{q} \in \mathscr{S}_{R}^{\prime}$ and

$$
\frac{\partial}{\partial q} W_{x}(q ; \mathbf{q})=\sum_{y \in R} W_{x, y}^{(1)}\left(q-q_{y}\right) .
$$

Proof. We have for $q>1$,

$$
W_{x, y}(2 q)-W_{x, y}(q)=\int_{q}^{2 q} d u W_{x, y}^{(1)}(u) .
$$

Therefore, since $W^{(1)}$ is increasing

$$
q W_{x, y}^{(1)}(q) \leqq W_{x, y}(2 q)-W_{x, y}(q) \leqq q W_{x, y}^{(1)}(2 q) .
$$

From this one gets for some $K>0$

$$
|q| \geqq 1 \Rightarrow\left|W_{x, y}^{(1)}(q)\right| \leqq K J(x, y)[1+|q|]^{n-1} .
$$

If $|q| \leqq 1$,

$$
\left|W_{x, y}^{(1)}(q)\right| \leqq \operatorname{Max}\left(W_{x, y}^{(1)}(1), W_{x, y}^{(1)}(-1)\right) \leqq 2^{n-1} K J(x, y)[1+|q|]^{n-1},
$$

and it is proved.

The same estimate suffices to prove ii).

Now to find a bound for III. 12, we shall expand the integral around the minimum of the exponent. Since it is $\mathscr{C}^{1}$ with respect to $q$, the absolute minimum $q_{c}$ is the solution of

$$
V^{(1)}\left(q_{c}\right)+\frac{\partial}{\partial q} W_{x}\left(q_{c} ; \mathbf{q}\right)=0
$$


Lemma III.4. (i) There is $0 \leqq \mathbf{q}_{0} \in \mathscr{S}^{\prime}$ such that if $\mathbf{q} \geqq \mathbf{q}_{0}$, the equation III.20 has a unique solution $q_{c}(\mathbf{q})$.

(ii) For $\mathbf{q} \geqq \mathbf{q}_{0}, q_{c}$ is an increasing unbounded function of $\mathbf{q}$.

Proof. Let $M$ be a positive real, and $J(M)$ be the set of $\mathbf{q} \in \mathscr{S}_{R}^{\prime}$, such that III. 20 has at least one solution $q_{c}$ greater than $M$. We claim that $J(M)$ is not empty.

III. 20 always has solutions because $V^{(1)}$ is asymptotically strictly increasing and unbounded, and $\frac{\partial W_{x}}{\partial q}$ is a strictly increasing function of $q$. On the other hand, if every solution of III. 20 is bounded by $M$, for every $\mathbf{q} \in \mathscr{S}_{R}^{\prime}, V^{(1)}\left(q_{c}\right)$ would remain bounded, whereas if $q_{y} \rightarrow \pm \infty, W_{x, y}^{(1)}\left(q_{c}-q_{y}\right)$ goes to $\pm \infty$, contradicting III. 20 .

Now let $M_{1} \geqq M_{0}$ (see Sect. I-B.) such that:

$$
\sup _{q \leqq M_{1}} V^{(1)}(q)=V^{(1)}\left(M_{1}\right) .
$$

$M_{1}$ exists by the assumption $B$ because if $|q| \geqq M_{0}, V^{(1)}(q)$ is strictly increasing. Therefore if

$$
a=\sup _{q \leqq M_{0}} V^{(1)}(q),
$$

it is sufficient to choose $M_{1}>M_{0}$ such that $V^{(1)}\left(M_{1}\right)>a$.

Now if $\mathbf{q} \in J\left(M_{1}\right)$, Eq. III. 20 has only one solution $q_{c} \geqq M_{1}$ because

$$
q \in\left[M_{0}, \infty\right] \Rightarrow V^{(1)}(q)+\frac{\partial}{\partial q} W(q ; \mathbf{q})
$$

is strictly increasing. On the other hand, if $q<M$, we have: (due to III. 21)

$$
V^{(1)}(q)+\frac{\partial}{\partial q} W(q ; \mathbf{q})<V^{(1)}\left(q_{c}\right)+\frac{\partial}{\partial q} W\left(q_{c} ; \mathbf{q}\right)=0 .
$$

Thus, if $\mathbf{q} \in J\left(M_{1}\right)$, III. 20 has exactly one solution (greater than $M_{1}$ ). Clearly $q_{c}$ is increasing in $\mathbf{q}$; indeed: let $\mathbf{q}^{\prime} \geqq \mathbf{q}, \mathbf{q}^{\prime} \neq \mathbf{q}$, then :

$$
V^{(1)}\left(q_{c}\right)+\frac{\partial}{\partial q} W\left(q_{c}, \mathbf{q}^{\prime}\right)<V^{(1)}\left(q_{c}\right)+\frac{\partial}{\partial q} W\left(q_{c}, \mathbf{q}\right)=0 .
$$

and if

$$
q \rightarrow+\infty V^{(1)}(q)+\frac{\partial}{\partial q} W\left(q, \mathbf{q}^{\prime}\right) \rightarrow+\infty .
$$

Therefore, there is $q_{c}^{\prime}>q_{c}$ such that

$$
V^{(1)}\left(q_{c}^{\prime}\right)+\frac{\partial}{\partial q} W\left(q_{c}^{\prime}, \mathbf{q}^{\prime}\right)=0
$$

Picking $\mathbf{q}_{0}$ in $J\left(M_{1}\right)$, we have proved our lemma.

Lemma III.5. Let $\mathbf{q}_{0}$ be as in the previous lemma. For $\mathbf{q} \geqq \mathbf{q}_{0}, \mathbf{q} \in \mathscr{S}^{\prime}$, we have for some $K_{1} \geqq 0$. 


$$
I(\mathbf{q})=\frac{\int_{q_{c}}^{\infty} d q \exp \left(-V(q)-W_{x}(q ; \mathbf{q})+a q\right)}{\int_{q_{c}}^{\infty} d q \exp \left(-V(q)-W_{x}(q ; \mathbf{q})\right)} \leqq K_{1} \exp \left(\frac{a^{2}}{2 A q_{c}^{\alpha}}+a q_{c}\right)
$$

Proof. Let $F$ be a function on $\mathbb{R}$ and define

$$
I_{F}=\frac{\int_{q_{c}}^{\infty} d q \exp (-F(q)+a q)}{\int_{q_{c}}^{\infty} d q \mathrm{e}^{-F(q)}} .
$$

We claim that if $F_{1}, F_{0}$ are two functions such that $F_{1}-F_{0}$ is increasing on $\left[q_{c}, \infty\right]$, then

$$
I_{F_{1}} \leqq I_{F_{0}} \text {. }
$$

For if $F_{\alpha}=\alpha F_{1}+(1-\alpha) F_{0}$, we find (with obvious notations)

$$
2 \frac{\partial I_{F_{\alpha}}}{\partial \alpha}=-\frac{1}{Z_{(\alpha)}^{2}} \int_{q_{c}}^{\infty} d q \mathrm{e}^{-F_{\alpha}}(q) \int_{q_{c}}^{\infty} d p \mathrm{e}^{-F_{\alpha}}\left[\mathrm{e}^{a q}-\mathrm{e}^{a p}\right]\left[\left(F_{1}-F_{0}\right)(q)-\left(F_{1}-F_{0}\right)(p)\right] .
$$

Since both $q \rightarrow \mathrm{e}^{a q}$ and $F_{1}-F_{0}$ are increasing on $\left[q_{c}, \infty\right]$, the integrand is positive, and the claim is proven.

Let us apply this remark to our case:

$$
\begin{aligned}
& F_{1}=V(q)+W_{2}(q ; \mathbf{q}), \\
& F_{0}=\frac{A}{(\alpha+1)(\alpha+2)}\left[q^{\alpha+2}-q_{c}^{\alpha+2}-\left(q-q_{c}\right) q_{c}^{\alpha+1}(\alpha+2)\right] . \quad \text { b) }
\end{aligned}
$$

Indeed, by definition of $q_{c}$ we have:

$$
\begin{aligned}
F_{1}^{(1)} & =V^{(1)}(q)+W_{x}^{(1)}(q ; \mathbf{q}) \geqq \int_{q_{c}}^{q} d u V^{(2)}(u) \\
& \geqq A \int_{q_{c}}^{q} d u u^{\alpha}=F_{0}^{(1)}, q \geqq q_{c},
\end{aligned}
$$

Because $V^{(2)}(u) \geqq A|u|^{\alpha}$ by $(B)$ for $u>q_{c}>M_{1}$ and

$$
W_{x}^{(1)}(q ; q)-W_{x}^{(1)}\left(q_{c} ; \mathbf{q}\right) \geqq 0 \quad q \geqq q_{c} .
$$

Thus $F_{1}-F_{0}$ is increasing on $\left[q_{c}, \infty\right]$. Now, replacing $F_{1}$ by $F_{0}$, and putting

$$
q=q_{c}+\frac{x}{q_{c}^{\alpha / 2}}
$$

we get (with $Z\left(q_{c}\right)$ a normalization factor)

$$
I(\mathbf{q}) \leqq \frac{\mathrm{e}^{a q_{c}}}{Z\left(q_{c}\right)} \int_{0}^{\infty} d x \exp \left(-A x^{2} \int_{0}^{1} d \theta(1-\theta)\left(1+\frac{x}{q_{c}^{\alpha / 2+1}}\right)^{\alpha}+a \frac{x}{q_{c}^{\alpha / 2}}\right)
$$


By the dominated convergence theorem, the normalization factor $Z\left(q_{c}\right)$ converges to:

$$
\lim _{q_{c} \rightarrow \infty} Z\left(q_{c}\right)=\int_{0}^{\infty} d x \exp \left(\frac{-A x^{2}}{2}\right)=\sqrt{2 \pi A},
$$

whereas the numerator is dominated by:

$$
\int_{0}^{\infty} d x \exp \left(\frac{-A x^{2}}{2}+a x / q_{c}^{\alpha / 2}\right)=\exp \left(a^{2} / 2 A q_{c}^{\alpha}\right)
$$

and the lemma is proved.

Due to III. 12 with $p_{0}=q_{c}$ and III. 28, Theorem III. 2 will be proved once the following holds:

Lemma III.6. The following estimate holds if $\mathbf{q} \in J\left(M_{1}\right)$

$$
q_{c} \leqq B+\sum_{y \in R} r(x, y)\left|q_{y}\right|
$$

where $B>0$ and $r$ is such that $\|\mathbf{r}\|<1,\|\mathbf{r}\|_{\eta, N}<+\infty \forall N \geqq 0$.

Proof. Let us assume $\alpha>n-2$. Then $q_{c}$ minimizes the function $q \rightarrow V(q)+$ $+\sum_{y \in R} W_{x, y}\left(q-q_{y}\right)$. Thus one gets

$$
V\left(q_{c}\right) \leqq V\left(q_{c}\right)+W_{x}\left(q_{c} ; \mathbf{q}\right) \leqq V(0)+\sum_{y} W_{x, y}\left(-q_{y}\right) .
$$

Using A1 - A2 one concludes

$$
V\left(M_{1}\right)+\left(q_{c}-M_{1}\right) V^{(1)}\left(M_{1}\right)+A \int_{M_{1}}^{q_{c}}\left(q_{c}-u\right) u^{\alpha} d u \leqq V(0)+\sum_{y} J(x, y)\left[1+\left|q_{y}\right|\right]^{n} .
$$

Changing eventually $A$ into $\frac{A}{2}$, we then can find a constant $B_{1}$ such that:

$$
q_{c}>M_{1} \Rightarrow \frac{A}{2(\alpha+1)(\alpha+2)} q_{c}^{\alpha+2} \leqq B_{1}+\sum_{y} J(x, y)\left[1+\left|q_{y}\right|\right]^{n} .
$$

Thus, there are constants $B_{2}, A_{2}$ such that

$$
q_{c} \leqq B_{2}+A_{2} \sum_{y} J(x, y)^{1 /(\alpha+2)}\left[1+\left|q_{y}\right|\right]^{n /(\alpha+2)}
$$

Since

$$
n<\alpha+2\left(1+\left|q_{y}\right|\right)^{n /(\alpha+2)} \leqq a(\varepsilon)+\varepsilon\left(1+\left|q_{y}\right|\right), \forall \varepsilon>0
$$

and thus III.39 is proved.

Let us assume A2 to hold. Then from III.20 we get

$$
V^{(2)}\left(q_{c}\right) \frac{\partial q_{c}}{\partial y}+\sum_{y} W_{x, y}^{(2)}\left(q_{c}-q_{y}\right) \frac{\partial q_{c}}{\partial q_{y}}=W_{x, y}^{(2)}\left(q_{c}-q\right)
$$


From $\mathrm{A} 2-B$ (taking $\alpha=0$ ) we get:

$$
\frac{\partial q_{c}}{\partial q_{y}} \leqq \frac{J(x, y)}{A+\sum_{y} J(x, y)}=r(x, y)
$$

from which III.39 follows with

$$
\|\mathbf{r}\|=\frac{\|\mathbf{J}\|}{A+\|\mathbf{J}\|}<1
$$

and

$$
\|\mathbf{r}\|_{\eta, N}=\frac{\|\mathbf{J}\|_{\eta, N}}{A+\|\mathbf{J}\|}<+\infty .
$$

\section{The FKG Order}

The configuration space $\Omega_{R}$ is ordered by

$$
\mathbf{q} \leqq \mathbf{q}^{\prime} \Leftrightarrow \mathbf{q}_{x} \leqq q_{x}^{\prime} \quad \forall x \in R .
$$

We denote $\mathscr{K}_{+}\left(\Omega_{R}\right)$ (respectively $\left.\mathscr{K}_{-}\left(\Omega_{R}\right)\right)$ the set of bounded increasing respectively decreasing) measurable functions on $\Omega_{R} \cdot \mathscr{K}_{+}\left(\Omega_{R}\right)$ is a convex cone; it determines the order in the following sense

$$
\mathbf{q} \leqq \mathbf{q}^{\prime} \Leftrightarrow F(\mathbf{q}) \leqq F\left(\mathbf{q}^{\prime}\right) \quad \forall F \in \mathscr{K}_{+}\left(\Omega_{R}\right) .
$$

Moreover, if $\mu_{1}, \mu_{2}$ are probability measures on $\Omega_{R}$ coinciding on $\mathscr{K}_{+}\left(\Omega_{R}\right), \mu_{1}=\mu_{2}$, for $\mathscr{K}_{+}\left(\Omega_{R}\right) \cap \mathscr{C}\left(\Omega_{R}\right)$ separates the points of $\Omega_{R}$, contains constants and is invariant by complex conjugacy. Thus, for every compact set $K \subset \Omega_{R}, \mathscr{C}\left(\Omega_{R}\right) \cap \mathscr{K}_{+}\left(\Omega_{R}\right)$ generates a dense ${ }^{*}$-algebra in $\mathscr{C}(K)$. Thus $\mu_{1}$ and $\mu_{2}$ coincide on compact subsets and this implies $\mu_{1}=\mu_{2}$.

A dual order can be defined on $\mathfrak{M}\left(\Omega_{R}\right)$ by

$$
\mu_{1} \propto \mu_{2} \Leftrightarrow \mu_{1}(F) \leqq \mu_{2}(F) \quad \forall F \in \mathscr{K}_{+}\left(\Omega_{R}\right) .
$$

It will be called the FKG order [40].

The remarkable property of our model comes from the fact that the local specification $\mathscr{E}$ leaves $\mathscr{K}_{+}\left(\Omega_{R}\right)$ invariant (see Prop. IV.2 below). Following Preston [40] we propose:

Definition IV.1. A local specification $\mathscr{E}=\left(E_{\Lambda^{c}}\right)_{\Lambda \in \mathscr{F}(R)}$ is called attractive, if for any $\Lambda \in \mathfrak{F}(R)$

$$
E_{A^{c}}\left(\mathscr{K}_{+}\left(\Omega_{R}\right)\right) \subset \mathscr{K}_{+}\left(\Omega_{R}\right)
$$

Proposition IV.2. The local specification I.5 is attractive, provided $\left(W_{x, y}\right)_{x, y}$ satisfies $A$.

Proof. Indeed, let $F$ be in $\mathscr{K}_{+}\left(\Omega_{R}\right)$, and $\mathbf{q} \leqq \mathbf{q}^{\prime}$. We get

$$
E_{\Lambda^{c}}^{\mathbf{q}}(F)=\int d \mu_{\Lambda}^{\mathbf{q}}\left(\mathbf{p}_{\Lambda}\right) F\left(\mathbf{p}_{\Lambda}, \mathbf{q}_{\Lambda^{c}}\right) .
$$


Replacing $F\left(\mathbf{p}_{A}, \mathbf{q}_{A^{c}}\right)$ by $F\left(\mathbf{p}_{A}, \mathbf{q}_{A^{c}}^{\prime}\right)$ inside the integral, gives an upper bound of the right hand side. On the other hand, the probability measure $\mu_{\Lambda}^{\mathrm{q}}$ has the form:

$$
d \mu_{\Lambda}^{\mathbf{q}}\left(\mathbf{p}_{\Lambda}\right)=\frac{1}{Z(\mathbf{q})} \prod_{x \in \Lambda}\left(d \mathbf{p}_{x} \exp \left(-V\left(p_{x}\right)-\sum_{y \neq \Lambda} W_{x, y}\left(p_{x}-q_{y}\right)\right) \exp \left(-\sum_{x, y \in \Lambda} W_{x, y}\left(p_{x}-p_{y}\right)\right) .\right.
$$

Since

$$
\begin{aligned}
& \frac{\partial^{2}}{\partial p_{x} \partial p_{y}}\left(-\sum_{x^{\prime} \in \Lambda}\left(V\left(p_{x^{\prime}}\right)+\sum_{y^{\prime} \in \Lambda} W_{x^{\prime} y^{\prime}}\left(p_{x^{\prime}}-q_{y^{\prime}}\right)\right)-\sum_{x^{\prime}, y^{\prime} \in \Lambda} W_{x^{\prime} y^{\prime}}\left(p_{x}-p_{y}\right)\right. \\
& =W_{x, y}^{(2)}\left(p_{x}-p_{y}\right) \geqq 0,
\end{aligned}
$$

it follows that $\mu_{A}^{\mathbf{q}}$ satisfies the FKG inequalities [43]

$$
\mu_{\Lambda}^{\mathbf{q}}\left(G_{1} G_{2}\right) \geqq \mu_{\Lambda}^{\mathbf{q}}\left(G_{1}\right) \mu_{\Lambda}^{\mathbf{q}}\left(G_{2}\right), \quad G_{1}, G_{2} \in \mathscr{K}_{+}\left(\Omega_{\Lambda}\right) .
$$

In particular if $G \in \mathscr{K}_{+}\left(\Omega_{\Lambda}\right)$ :

$$
y \notin \Lambda \Rightarrow \frac{\partial}{\partial q_{y}} \mu_{\Lambda}^{\mathbf{q}}(G)=\sum_{x \in \Lambda}\left\{\mu_{\Lambda}^{\mathbf{q}}\left(G W_{x, y}^{(1)}\left(\sigma_{x}-q_{y}\right)\right)-\mu_{\Lambda}^{\mathbf{q}}(G) \mu_{\Lambda}^{\mathbf{q}}\left(W_{x, y}\left(\sigma_{x}-q_{y}\right)\right)\right\}
$$

is positive. Thus:

$$
\mu_{\Lambda}^{\mathbf{q}}(G) \leqq \mu_{\Lambda}^{\mathbf{q}^{\prime}}(G), \quad G \in \mathscr{K}_{+}\left(\Omega_{\Lambda}\right) .
$$

If $G\left(\mathbf{p}_{A}\right)=F\left(\mathbf{p}_{A}, \mathbf{q}_{A}^{\prime}\right)$ we get the result:

$$
E_{\Lambda^{c}}^{\mathbf{q}}(F) \leqq \mu_{\Lambda}^{\mathbf{q}}(G) \leqq \mu_{\Lambda}^{\mathbf{q}^{\prime}}(G)=E_{\Lambda^{c}}^{\mathbf{q}^{\prime}}(F) .
$$

The main property of local compact attractive specifications is described in the following:

Theorem IV.3. Let $\mathscr{E}$ be a local, compact attractive specification. Then $\mathscr{G}_{t}(\mathscr{E})$ has a unique maximum $\mu_{+}$and a unique minimum $\mu_{-}$for the $\mathrm{FKG}$ order.

Proof. First we prove the existence of a maximum. Let $\left(\mu_{\alpha}\right)_{\alpha \in A}$ be a totally ordered net in $G_{t}(\mathscr{E})$. Since $\mathscr{E}$ is compact, it has a limit point $\mu_{\infty}$ in $\mathscr{G}_{t}(\mathscr{E})$. In particular there is an increasing subnet $\alpha(\beta)$ such that

$$
\mu_{\infty}(F)=\lim _{\beta} \mu_{\alpha(\beta)}(F), \quad \forall F \in \mathscr{K}_{+}\left(\Omega_{R}\right) .
$$

Since $\beta \rightarrow \mu_{\alpha(\beta)}$ is increasing in the FKG order we get:

$$
\mu_{\infty}(F)=\sup _{\alpha} \mu_{\alpha}(F) \quad F \in \mathscr{K}_{+}\left(\Omega_{R}\right) .
$$

Thus $\mu_{\infty}$ is unique since it is uniquely defined on $\mathscr{K}_{+}\left(\Omega_{R}\right)$, and is precisely the supremum of $\left(\mu_{\alpha}\right)_{\alpha \in A}$. By the Zorn lemma, $\mathscr{G}_{t}(\mathscr{E})$ has maximal elements.

Let now $\mu_{1}$ and $\mu_{2}$ be two such maximal elements in $\mathscr{G}_{t}(\mathscr{E})$. In $\Omega_{R}$, the map

$$
\left(\mathbf{q}, \mathbf{q}^{\prime}\right) \in \Omega_{R} \times \Omega_{R} \rightarrow \mathbf{q} \vee \mathbf{q}=\left(\max \left(q_{x}, q_{x}^{\prime}\right)\right)_{x \in R} \in \Omega_{R}
$$


is measurable. Let $\rho$ be the image of $\mu_{1} \otimes \mu_{2}$ under this map. We claim that: $\rho \in \mathfrak{M}_{t}$. For

$$
\begin{aligned}
\rho\left(\left|\sigma_{x}\right|\right) & =\int d \mu_{1}(\mathbf{q}) d \mu_{2}(\mathbf{p})\left|q_{x} \vee p_{x}\right| \\
& \leqq \int d \mu_{1}(\mathbf{q}) d \mu_{2}(\mathbf{p})\left(\left|q_{x}\right|+\left|p_{x}\right|\right) \leqq \mu_{1}\left(\left|\sigma_{x}\right|\right)+\mu_{2}\left(\left|\sigma_{x}\right|\right) .
\end{aligned}
$$

On the other hand, $\rho>\mu_{i}, i=1,2$, because:

$$
\begin{aligned}
F \in \mathscr{K}_{+}\left(\Omega_{R}\right) & \Rightarrow \rho(F)=\int d \mu_{1}(\mathbf{q}) d \mu_{2}(\mathbf{p}) F(\mathbf{q} \vee \mathbf{p}) \\
& \geqq \int d \mu_{i}(\mathbf{q}) F(\mathbf{q}), \quad i=1,2 .
\end{aligned}
$$

Since $\mathscr{E}$ is attractive, it follows that

$$
i=1,2, \quad \mu_{i}=\mu_{i} E_{A^{c}} \propto \rho E_{\Lambda^{c}} \quad \forall \Lambda \in \mathfrak{F}(R) .
$$

Since $\mathscr{E}$ is compact, $\left(\rho E_{A^{c}}\right)_{\Lambda \in \widetilde{F}(R)}$ is compact in $\mathfrak{M}_{t}$, and any limit point $\rho_{\infty}$ is a tempered Gibbs state such that

$$
\mu_{1} \propto \rho_{\infty}, \mu_{2} \propto \rho_{\infty} .
$$

By definition of $\mu_{1}, \mu_{2}$ we get

$$
\mu_{1}=\mu_{2}=\rho_{\infty} .
$$

The previous theorem is too abstract. The following result gives a rather concrete construction of $\mu_{+}$.

Proposition IV.4. With the hypothesis of Theorem IV.3, the maximal state $\mu_{+}$is given by:

$$
\mu_{+}=\lim _{\Lambda \uparrow R} E_{\Lambda^{c}}^{\mathbf{q}}
$$

for any $\mathbf{q} \in \mathscr{S}_{R}^{\prime}$ such that

i) $q_{x}>0 \quad \forall x$.

ii) $\exists N>0$ depending only on $\mathscr{E}$, such that

$$
\sum_{x \in R} \frac{d(0, x)^{N}}{q_{x}}<+\infty .
$$

If moreover $\mathscr{E}$ satisfies the exponential bound III.1, then, ii) can be replaced by iia)

$$
q_{x} \geqq B \log |d(0, x)|^{1 / 2}
$$

for B large enough.

Remark. The estimate IV.22 has already been proposed by J. Lebowitz and E. Presutti [31].

Proof. For $\mathbf{q} \in \mathscr{S}_{R}^{\prime}$, the Dirac measure $\delta_{\mathbf{q}}$ belongs to $\mathfrak{M}_{t}\left(\Omega_{R}\right)$ and $E_{A^{c}}^{\mathbf{q}}=\delta_{\mathbf{q}} \cdot E_{A^{c}} \cdot \mathscr{E}$ being compact, this family of measure has a limit point $\mu_{\infty}$.

Now, let $F$ be in $\mathscr{K}_{+}\left(\Omega_{R}\right)$, and $\mu$ be in $\mathscr{G}_{t}(\mathscr{E})$ :

$$
\mu(F)=\int_{\mathbf{p}_{\Lambda^{c} \subseteq \mathbf{q}_{\Lambda^{c}}}} d \mu(\mathbf{p}) E_{A^{c}}^{\mathbf{p}}(F)+\int_{\mathbf{p}_{A c} \geqq \mathbf{q}_{A^{c}}} d \mu(\mathbf{p}) E_{A^{c}}^{\mathbf{p}}(F) .
$$


Since $\mathscr{E}$ is attractive, $E_{\Lambda^{c}}^{\mathbf{p}}(F)$ is an increasing function of $\mathbf{p}$; on the other hand $F$ is bounded by $\|F\|_{\infty}$. Thus:

$$
\mu(F) \leqq E_{\Lambda^{c}}^{\mathbf{q}}(F)+\|F\|_{\infty} \mu\left\{\mathbf{p} \in \Omega_{R} ; \exists x \notin \Lambda, p_{x} \geqq q_{x}\right\} .
$$

The last term of the r.h.s. can be estimated by:

$$
\sum_{x \notin \Lambda} \frac{\mu\left(\left|\sigma_{x}\right|\right)}{q_{x}} \leqq \sum_{x \in \Lambda^{c}} \frac{K d(O, x)^{N}}{q_{x}}=\varepsilon_{\Lambda}
$$

where $K>0$, and $N \geqq 0$ depends only on $\mathscr{E}$ since $\mathscr{G}_{t}(\mathscr{E})$ is a compact subset of $\mathfrak{M}_{t}\left(\Omega_{R}\right)$. By the hypothesis for any sequence $\Lambda_{n}$ "converging to $R, \lim \varepsilon_{\Lambda_{n}}=0$. Choosing a suitable subsequence $\Lambda_{n}$ we get:

$$
\mu(F) \leqq \mu_{\infty}(F) \quad F \in \mathscr{K}_{+}\left(\Omega_{R}\right)
$$

which proves that $\mu_{\infty}=\mu_{+}$.

Now if $\mathscr{E}$ satisfies the exponential bound IV.25 can be replaced by (see eq. III.3)

$$
\sum_{x \in \Lambda^{c}} \inf \left[\mu\left(\exp \left(a \sigma_{x}\right) \exp \left(-a q_{x}\right)\right] \leqq \sum_{x \in \Lambda^{c}} \exp \left(\frac{-\left(q_{x}-B^{\prime}\right)^{2}}{2 A^{\prime}}\right)\right. \text {. }
$$

Since $R \subset \mathbb{Z}^{v}$ the r.h.s. converges provided IV.22 holds with

$$
\frac{B^{\prime 2}}{2 A^{\prime}}>v
$$

\section{The General Properties of $\boldsymbol{\mu}_{ \pm}$}

The remarkable states $\mu_{ \pm}$may have inherited properties of the local specification. In this section we prove that $\mu_{ \pm}$are extremal in $\mathscr{G}_{t}(\mathscr{E})$ and that $\mathscr{G}_{t}(\mathscr{E})$ is reduced to one point if and only if $\mu_{+}=\mu_{-}$. We prove that $\mu_{ \pm}$are translation invariant if $\mathscr{E}$ is translation covariant. They are reflection positive under suitable properties of $\mathscr{E}$. At last, we prove that they have the global Markov property if $\mathscr{E}$ is locally Markov. In what follows $\mathscr{E}$ is a local compact, attractive specification.

\section{V.a Extremality}

Proposition V.1. The states $\mu_{+}$and $\mu_{-}$are extremal in $\mathscr{G}_{t}(\mathscr{E})$ and $\mathscr{G}_{t}(\mathscr{E})$ has one point if and only if $\mu_{+}=\mu_{-}$.

Proof. Assume $\mu_{+}=\alpha \mu_{1}+(1-\alpha) \mu_{2}$ with $0<\alpha<1, \mu_{i} \in \mathscr{G}_{t}(\mathscr{E})$. For $F$ belonging to $\mathscr{K}_{+}\left(\Omega_{R}\right)$ we get

$$
\begin{aligned}
& \mu_{+}(F)=\alpha \mu_{1}(F)+(1-\alpha) \mu_{2}(F) \\
& \mu_{+}(F) \geqq \mu_{i}(F), \quad i=1,2 .
\end{aligned}
$$

Thus

$$
\mu_{+}(F)=\mu_{1}(F)=\mu_{2}(F), \quad \forall F \in \mathscr{K}_{+}\left(\Omega_{R}\right)
$$


which means

$$
\mu_{+}=\mu_{1}=\mu_{2} \text {. }
$$

If moreover $\mu_{+}=\mu_{-}$we have by construction:

$$
\mu_{+}(F)=\mu_{-}(F) \leqq \mu(F) \leqq \mu_{+}(F) \quad \forall F \in \mathscr{K}_{+}\left(\Omega_{R}\right) \quad \forall \mu \in \mathscr{G}_{t}(\mathscr{E})
$$

That is

$$
\mu=\mu_{+}=\mu_{-} \quad \forall \mu \in \mathscr{G}_{t}(\mathscr{E}) .
$$

V.b Covariance. Let $\tau$ be a Borel isomorphism of $\Omega_{R}$ into itself. For $F$ a measurable function, we put

$$
\tau F(\mathbf{q})=F\left(\tau^{-1} \mathbf{q}\right)
$$

Definition $V .2 . \mathscr{E}$ is said to be $\tau$-covariant if for any $\Lambda \in \mathfrak{F}(R)$ there is $\Lambda_{\tau} \in \mathfrak{F}(R)$ such that

$$
\tau E_{\Lambda^{\mathrm{c}}}=E_{\Lambda_{\tau}^{\mathrm{c}}} \tau
$$

Proposition V.3. Let $\mathscr{E}$ be $\tau$-covariant. Then $\mathscr{G}_{t}(\mathscr{E})$ is $\tau$-invariant. Moreover if the action $\tau$ respects the order (i.e. $\left.\tau^{ \pm 1} \mathscr{K}_{+}\left(\Omega_{R}\right) \subset \mathscr{K}_{+}\left(\Omega_{R}\right)\right)$ then $\mu_{+}$and $\mu_{-}$are $\tau$ invariant. If $\tau$ reverses the order (i.e. $\left.\tau^{ \pm 1} \mathscr{K}\left(\Omega_{R}\right) \subset \mathscr{K}_{-}\left(\Omega_{R}\right)\right), \tau^{*} \mu_{+}=\mu_{-}$.

Proof. Let $\mu$ be a tempered Gibbs state. Then $\tau^{*} \mu$ satisfies for every $F$ bounded and measurable and $\Lambda \in \mathfrak{F}(R)$

$$
\tau^{*} \mu(F)=\mu(\tau F)=\mu\left(E_{\Lambda_{\tau}^{c}}(\tau F)\right)=\mu\left(\tau E_{A^{c}}(F)\right)=\tau^{*} \mu E_{\Lambda^{c}}(F) .
$$

Thus

$$
\tau^{*} \mathscr{G}_{t}(\mathscr{E}) \subset \mathscr{G}_{t}(\mathscr{E})
$$

Since $\mu_{+}$is maximal we get:

$$
\mu_{-} \propto \tau^{*} \mu_{+} \propto \mu_{+} \quad \mu_{-} \propto \tau^{-1 *} \mu_{+} \propto \mu_{+} .
$$

If moreover $\tau^{*}$ respects the order we get from V.10:

$$
F \in \mathscr{K}_{+}\left(\Omega_{R}\right) \Rightarrow \mu_{+}(F)=\tau^{-1 *} \mu_{+}(\tau F) \leqq \mu_{+}(\tau F)=\tau^{*} \mu_{+}(F) \leqq \mu_{+}(F),
$$

which means:

$$
\mu_{+}=\tau^{*} \mu_{+}
$$

If $\tau^{*}$ reverses the order, $F$ is decreasing and

$$
F \in \mathscr{K}_{+}\left(\Omega_{R}\right) \Rightarrow \mu_{-}(F)=\tau^{-1 *} \mu_{-}(\tau F) \geqq \mu_{+}(\tau F)=\tau^{*} \mu_{+}(F) \geqq \mu_{-}(F) .
$$

Thus

Examples. (i)

$$
\mu_{-}=\tau^{*} \mu_{+} .
$$

$$
R=\mathbb{Z}^{v} \quad(\tau q)_{x}=q_{x+a} \quad a \in \mathbb{Z}^{v} .
$$


The local specification in I.5 is translation invariant

iff

$$
W_{(x, y)}(q)=W_{((x-y), 0)}(q), \quad \forall x, \forall y
$$

(translation invariant interaction). Then $\tau$ respects the order.

ii)

$$
\tau \mathbf{q}=-\mathbf{q} \quad \text { (spin flip). }
$$

The local specification is $\tau$-invariant iff it is even, which means

$$
V(-q)=V(q) \quad W_{x, y}(-q)=W_{x, y}(q) \quad \forall x, y \in R .
$$

In this case, $\tau$ reverses the order.

V.c Reflection Positivity. Let us consider the case $R=\mathbb{Z}^{v}$. Let $\Pi$ be the hyperplane $\Pi=\left\{x \in \mathbb{R}^{v}, x_{1}=\frac{1}{2}\right\}$, and $\theta$ be the reflection about $\Pi$. $\theta$ leaves $\mathbb{Z}^{v}$ invariant and exchanges the half spaces $\Pi_{+}$and $\Pi_{-}$defined by $\Pi$ in $\mathbb{Z}^{v}$. Let $\Theta$ be the corresponding action on the bounded measurable function on $\Omega_{R}$ :

$$
\Theta F(\mathbf{q})=F(\theta \mathbf{q}) \quad \text { with } \quad(\theta \mathbf{q})_{x}=q_{\theta x} \quad x \in \mathbb{Z}^{v} .
$$

We denote by $\mathscr{P}$, the closed convex cone generated by $\overline{\Theta F} F$ where $F$ is bounded and $\mathscr{B}_{\Pi_{+}}$-measurable. (We take the closure with respect to the topology induced by weak topology in $\bigcap_{\mu \in \mathfrak{M}_{t}} L^{\infty}\left(\Omega_{R}, \mathscr{B}, \mu\right)$ for every tempered measure $\mu$ ).

Definition $V .4 . \mathscr{E}$ is reflection positive, if for any finite $\theta$-invariant subset $\Lambda$ of $R=\mathbb{Z}^{v}$, and any $\theta$-invariant configuration $\mathbf{q}, E_{A^{c}}^{\mathbf{q}}(F) \geqq 0, F \in \mathscr{P}_{+}$.

Proposition V.5. If $\mathscr{E}$ is reflection positive, $\mu_{+}$and $\mu_{-}$are reflection positive i.e.:

$$
\mu_{ \pm}(G) \geqq 0 \quad \forall G \in \mathscr{P}_{+} \cdot
$$

Proof. Let $\mathbf{q}$ be in $\mathscr{S}_{R}^{\prime}$ such that $\theta \mathbf{q}=\mathbf{q}$. Then $G(\mathbf{q}) \geqq 0 \forall G \in \mathscr{P}_{+}$, by definition of $\mathscr{P}_{+}$.

In particular if $q_{x} \geqq 0$ satisfies IV.21, and $\theta \mathbf{q}=\mathbf{q}$, then for $G$ being in $\mathscr{P}_{+}$we have:

$$
\mu_{+}(G)=\lim _{\Lambda} E_{\Lambda^{c}}^{\mathbf{q}}(G) \geqq 0 .
$$

Example. Let us consider the model I.5 with the following restrictions:

RP1) $W_{x, y}=0$ unless $d(x, y)=1$.

RP2) $W_{\theta x, \theta y}=W_{x, y} \quad x, y \in R$.

$\mathrm{RP} 3) \exp -W_{x, y}(q)$ is a function of positive type.

Proposition V.6. If the W's obey RP1-3), then the local specification in 1.5 is reflection positive.

Proof. Let $\Lambda$ be a finite subset of $\mathbb{Z}^{v} \theta$-invariant and $\Lambda_{ \pm}=\Lambda \cap \Pi_{ \pm}$. We claim that if $x \in \Lambda_{ \pm}, y \in \mathbb{Z}^{v}$ are such that $d(x, y)=1$, then either $y \in \Pi_{+}$, or $y=\theta x$. For 
if $y \notin \Pi_{+}$, we have

$$
d(x, y)=\left|x_{1}-y_{1}\right|+\sum_{\alpha=2}^{v}\left|x_{\alpha}-y_{\alpha}\right|=1
$$

and

$$
x_{1} \geqq 1 \quad y_{1} \leqq 0
$$

Thus

$$
\left|x_{1}-y_{1}\right|=1 \quad x_{\alpha}=y_{\alpha} \Rightarrow y=\theta x \text {. }
$$

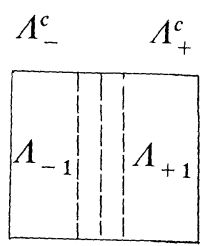

Let $\partial \Lambda_{+}$(respectively $\left.\partial \Lambda_{-}\right)$be the set of $x \in \Lambda_{+}$(respectively $\Lambda_{-}$) such that $d(x, \theta x)=1$; we put $\Lambda_{+}^{c}=\Pi_{+} \backslash_{+}$(respectively $\Lambda_{-}=$ $\left.\Pi_{-} \backslash \Lambda_{-}\right) \Lambda_{+, 1}=\Lambda_{+} \partial \Lambda_{+}$(respectively $\left.\Lambda_{-, 1}=\Lambda_{-} \backslash \partial \Lambda_{-}\right)$.

$\overleftrightarrow{\partial \Lambda_{-,}} \stackrel{\leftrightarrow}{\partial \Lambda_{+}}$

Then if $F$ is $\mathscr{B}_{\Pi_{1}}$-measurable and bounded

$$
\begin{aligned}
& E_{\Lambda^{c}}^{\mathbf{q}}(\Theta F . F)= \\
& \begin{array}{l}
\frac{1}{Z(\mathbf{q})} \int d \mu_{\Lambda_{+, 1}}\left(\mathbf{p}_{\Lambda_{+, 1}}\right) d \mu_{\Lambda_{+, 1}}\left((\theta \mathbf{p})_{\Lambda_{+, 1}}\right) \underset{x \in \partial \Lambda_{+}}{\Pi} d p_{x} d_{\theta x} \exp \left(-V\left(p_{x}\right)-\right. \\
\left.\quad-V\left(p_{\theta x}\right)-W_{x, \theta(x)}\left(p_{x}-p_{\theta x}\right)\right) \Theta \bar{F}_{W} F_{W}\left(\mathbf{p}_{\Lambda}, \mathbf{q}_{\Lambda^{c}}\right),
\end{array}
\end{aligned}
$$

where $d \mu_{\Lambda+Z}$ is some measure on $\Omega_{\Lambda_{+, 1}}, Z(\mathbf{q})$ a normalization factor, and:

$$
F_{W}\left(\mathbf{p}_{\Lambda}, \mathbf{q}_{\Lambda^{c}}\right)=F\left(\mathbf{p}_{\Lambda}, \mathbf{q}_{\Lambda_{+}^{c}}\right) \exp \left(-\sum_{x \in_{\Lambda_{+}}} \sum_{y \in \Lambda_{+}^{c}}\right) W_{x, y}\left(p_{x}-q_{y}\right)
$$

The numerator in V.25 can be written as:

$$
\int \prod_{x \in \partial \Lambda_{+}} d p_{x} d p_{\theta x} \exp \left(-W_{x, \theta x}\left(p_{x}-p_{\theta x}\right)\right) \hat{F}\left(p_{x}, \mathbf{q}_{\Lambda_{+}}\right) \hat{F}\left(p_{\theta x},(\theta \mathbf{q})_{\Lambda_{+}^{c_{+}}}\right)
$$

for some $\hat{F}$. Since $\exp \left(-W_{x, \theta x}(\mathbf{q})\right)$ is of positive type, we get a function in $\mathscr{P}_{+}$, because

$$
\sum_{0 \leqq 1, j \leqq N} M_{i j} \Theta \bar{F}_{i} F_{j} \in \mathscr{P}_{+} \text {if }\left(M_{i j}\right)_{i j} \geqq 0 .
$$

The same is true for $Z(\mathbf{q})$. Since $\mathbf{q}=\theta \mathbf{q}$ and $F \in \mathscr{P}{ }_{+} \Rightarrow F(\mathbf{q}) \geqq 0$, we get the result.

Remark. RP3 is verified by any function $W$ given by the Levy-Kintchine formula [51]

$$
W(q)=a q^{2}+b q+c-\int_{|s| \leqq 1}\left(\mathrm{e}^{i q s}-1-i q s\right) d v(s)-\int_{|s| \geqq 1} \mathrm{e}^{i q s} d v(q)
$$


where $v$ is a positive measure with:

$$
\int_{|s| \geqq 1} d v(s)+\int_{|s| \leqq 1} s^{2} d v(s)<+\infty .
$$

If we want $W$ to satisfy the assumption $A$, it is enough to assume $\int_{-\infty}^{+\infty} s^{2} d v(s)<+\infty$, $a \geqq 0$ and $s^{2} d v(s)$ is a measure of positive type. For example

$$
W(q)=a|q|^{\sigma}, \quad 1 \leq \sigma \leqq 2
$$

satisfies RP3.

V.d: The Markov Property. A measure $\mu$ has the Markov property if for every $F$, $\mathscr{B}_{\Lambda^{-}}$-measurable, $E_{\mu}\left(F \mid \mathscr{B}_{A^{c}}\right)$ depends only on the "boundary" of $\Lambda$; we will distinguish between the global Markov property for which no constraint is imposed on $\Lambda$, and the local one for which $\Lambda \in \mathfrak{F}(R)$ is needed. To be more precise we adopt the following definition $[1,2,3]$ :

Definition $V .7 .1^{\circ}$ ) A local specification $\mathscr{E}$ is locally Markov if one can find an integer $r \geqq 1$, such that for any finite subsets $\Lambda_{1}, \Lambda_{2}$ of $R$, with dist $\left(\Lambda_{1}, \Lambda_{2}\right)>r$, and any function $F_{i}(i=1,2) \mathscr{B}_{A_{i}}$-measurable one has:

$$
E_{\Lambda_{1} \cup \Lambda_{2}{ }^{c}}\left(F_{1} \cdot F_{2}\right)=E_{\left(\Lambda_{1} \cup \Lambda_{2}\right)^{c}}\left(F_{1}\right) E_{\left(\Lambda_{1} \cup \Lambda_{2}\right)^{c}}\left(F_{2}\right) .
$$

$2^{\circ}$ ) A tempered measure is locally Markov if the specification $\mathscr{E}_{\mu}=$ $\left(E_{\mu}\left(\cdot \mid \mathscr{B}_{\Lambda^{c}}\right)\right)_{\Lambda \in \mathfrak{F}(R)}$ is locally Markov. It is globally Markov if V.32 holds for $\mathscr{E}_{\mu}$, for any subset $\Lambda_{1}, \Lambda_{2}$ of $R$ with dist $\left(\Lambda_{1}, \Lambda_{2}\right)>r$.

The main result of this section is described below.

Theorem V.8. Let $\mathscr{E}$ be a locally Markov, local, compact attractive specification. Then, if $\mathscr{E}$ satisfies the Dobrushin estimate II.3, $\mu_{+}$and $\mu_{-}$have the global Markov property.

Proof. The main property of $\mathscr{E}$ is given by Lemma V.9 below. Once it is proved, the theorem follows trivially:

Lemma V.9. Let $\mathscr{E}$ as before, and $\mathcal{O} \subset R$. Then

$$
E_{\mu_{+}}^{\mathbf{q}}\left(F \mid \mathscr{B}_{\mathscr{O}^{c}}\right)=\lim _{\Lambda>R} E_{(\Lambda \cup \mathcal{O})^{c}}^{\mathbf{q}}(F)
$$

for measurable function $F$, and every $\mathbf{q} \in \mathscr{S}^{\prime}$ such that

$q_{x} \geqq 0 x \in \mathcal{O}, \sum_{x \in \theta} \frac{d(0, x)^{N}}{q_{x}}<+\infty$ for some $N$.

Proof. Let $\mathbf{p}$ be a slowly increasing sequence on $\mathcal{O}^{c}, \mathbf{p} \in \mathscr{S}_{\mathscr{O} c}^{\prime}$, and let $\mathscr{E}_{\mathbf{p}, \mathcal{O}}$ be the local specification on $\Omega_{\mathscr{O}}$ defined by:

$$
\mathscr{E}_{\mathbf{p}, \mathscr{O}}=\left(\hat{E}_{\Lambda^{c}}^{\mathbf{p}}\right)_{\Lambda \in \widetilde{\Im}(\mathcal{O})}
$$

with

$$
\hat{E}_{\Lambda^{c}}^{\mathbf{p}}(F)(\mathbf{q})=\left.E_{\Lambda^{c}}(F)\right|_{\left\{q_{x}=p_{x} ; x \notin \mathbb{O}\right\}} .
$$


In other words, the configurations coincide with $\mathbf{p}$ outside $\mathcal{O}$. Since the Dobrushin estimate holds for $\mathscr{E}$ we get

$$
x \in \mathcal{O} \hat{E}_{\{x\}^{\mathrm{c}}}^{\mathrm{p}}\left(\left|\sigma_{x}\right|\right) \leqq A_{x}+\sum_{y \notin \mathcal{O}} r(x, y)\left|p_{y}\right|+\sum_{y \in \mathcal{O}} r(x, y)\left|\sigma_{y}\right| .
$$

$\mathbf{p}=\left(p_{y}\right)_{y \in \mathscr{O}^{c}}$ being slowly increasing, $\|\mathbf{r}\|<1,\|\mathbf{r}\|_{\eta, N}<\infty$ implies that $\mathscr{E}_{\mathbf{p}, \mathscr{O}}$ obeys a Dobrushin estimate with

$$
A_{x}(\mathbf{p})=A_{x}+\sum_{y \notin \mathcal{C}} r(x, y)\left|p_{y}\right| .
$$

By virtue of Theorem II.3, $\mathscr{E}_{\mathbf{p}, \mathcal{O}}$ is a compact specification. It is also attractive if $\mathscr{E}$ is, as can be trivially verified from V.35. From Theorem IV.3, there is a maximal probability measure, say $M_{\mathbf{p}, \mathcal{O}}^{+}$on $\mathscr{G}_{t}\left(\mathscr{E}_{\mathbf{p}, \mathcal{O}}\right)$. From Proposition IV.4

$$
M_{\mathbf{p}, \mathcal{U}}^{+}(F)=\lim _{\Lambda \rightarrow \mathscr{O}} E_{\Lambda^{c}}^{\mathbf{p}, \mathbf{q}}(F), \quad \mathbf{p} \in \mathscr{S}_{\mathscr{O} \mathfrak{c}}^{\prime},
$$

where $\mathbf{q} \in \mathscr{S}_{\mathscr{O}}^{\prime}, q_{x} \geqq 0$ and $\sum_{x \in \mathcal{O}} \frac{d(0, x)^{N}}{q_{x}}<+\infty$ for some $N$ depending only on $\mathscr{E}$ and p. Thus $\mathbf{p} \rightarrow M_{\mathbf{p}, \mathcal{O}}^{+}(F)$ is $\mathscr{B}_{\mathscr{O} c} \underset{x \in \mathcal{O}}{ }$-measurable.

Now let $G$ be a $\mathscr{B}_{0 c}$-measurable function and $\mathbf{q}$ be in $\mathscr{S}_{R}^{\prime}$ as in Prop. IV.6

$$
\begin{aligned}
\mu_{+}(G F) & =\lim _{\Lambda>R} E_{\Lambda^{c}}^{\mathbf{q}}(F G)=\lim _{\Lambda>R} E_{\Lambda^{c}}^{\mathbf{q}}\left(E_{(\Lambda \cap \Theta) c}(G F)\right) \\
& =\lim _{\Lambda>R} E_{\Lambda^{c}}^{\mathbf{q}}\left(G \hat{E}_{\sigma_{\sigma^{c}}}(F)\right),
\end{aligned}
$$

where $\sigma_{\mathscr{0}}(x)=\sigma_{x}$ if $x \in \mathcal{O}^{c}$. Due to $V .38$ we get

$$
\mu_{+}(G F)=\mu_{+}\left(G M_{\sigma_{0} c, \theta}^{+}(F)\right) \text {. }
$$

Since $M_{\sigma_{\mathscr{O}}^{c}, \mathcal{O}}^{+}(F)$ is $B_{\mathscr{O}_{c}^{c}}$ measurable, the definition of the conditional expectation gives:

$$
E_{\mu_{+}}\left(F \mid \mathscr{B}_{\omega^{c}}\right)=M_{\sigma_{\sigma^{c},}, \Theta}^{+}(F)
$$

Examples. If the W's satisfy A with

$$
W_{x y}=0 \text { for } d(x, y)>r,
$$

then the model has the local Markov property.

\section{The Pressure and Uniqueness Criteria}

VI.a The Pressure. Coming back to the model I.5, we define the pressure with "free boundary condition" as follows:

$$
Z_{\Lambda}^{f}=\int \prod_{x \in \Lambda}\left(d q_{x} \exp \left(-V\left(q_{x}\right)\right)\right) \exp \left(-\sum_{x \in \Lambda} \sum_{y \in \Lambda} W_{x, y}\left(q_{x}-q_{y}\right)\right)
$$

and

$$
p^{f}=\lim _{\Lambda \rightarrow R}-\frac{1}{|\Lambda|} \log Z_{\Lambda}^{f}
$$


Here $|\Lambda|$ denotes the number of points in $\Lambda$, and the limit $\Lambda \nearrow R$ is taken in the van-Hove sense [44].

Proposition VI.1. If $V$ and $W$ 's satisfy $A, B$ then $p^{f}$ exists.

Proof. Without loss of generality we can normalize $V(q)$ such that

$$
\int d q \exp (-V(q))=1 \text {. }
$$

Then, since $W_{x, y}>0$ we get

$$
Z_{\Lambda}^{f} \leqq 1
$$

From the Jensen inequality we get

$$
Z_{\Lambda}^{f} \geqq \exp -\sum_{x, y \in \Lambda} \int d q d p \exp (-V(p)-V(q)) W_{x, y}(p-q) .
$$

Let us put

$$
a=\int d q d p \exp (-V(p)-V(q))[1+|p-q|]^{n} .
$$

It follows from $B$ that $0<a<+\infty$. Thus:

$$
Z_{\Lambda}^{f} \geqq \exp -a \sum_{x, y \in \Lambda} J(x, y) \geqq \exp -|\Lambda| a\|\mathbf{J}\| .
$$

On the other hand if $\Lambda_{1}, \Lambda_{2}$ are disjoint, $W_{x, y} \geqq 0$ implies

$$
Z_{\Lambda_{1} \cup A_{2}}^{f} \leqq Z_{\Lambda_{1}}^{f} Z_{\Lambda_{2}}^{f} \text {. }
$$

This estimate together with VI.4, VI.7, implies the result $[46,47]$.

Now, let $\mathbf{q}$ be in $\mathscr{S}_{R}^{\prime}$ and we define

$$
\begin{gathered}
Z_{\Lambda}(\mathbf{q})=\int \prod_{x \in \Lambda} d p_{x} \exp \left(-V\left(p_{x}\right)\right) \exp \left(-\sum_{x \in \Lambda} \sum_{y \in \Lambda} W_{x, y}\left(p_{x}-p_{y}\right)\right) \\
\cdot \exp \left(-\sum_{x \in \Lambda} \sum_{y \notin \Lambda} W_{x, y}\left(p_{x}-q_{y}\right)\right), \\
p_{\Lambda}(\mathbf{q})=-\frac{1}{|\Lambda|} \log Z_{\Lambda}(\mathbf{q}),
\end{gathered}
$$

and

$$
p^{\mu}=\lim _{\Lambda>R} \int d \mu(\mathbf{q}) p_{\Lambda}(\mathbf{q}), \mu \in \mathfrak{M}_{t}\left(\Omega_{R}\right) .
$$

Proposition VI.2. Let us assume $A, B$. If $\mu \in \mathscr{G}_{t}(\mathscr{E})$ then $p^{\mu}$ exists and coincides with $p^{f}$, provided there is $\varepsilon>0$ such that $\frac{|\partial \Lambda|}{|\Lambda|^{1-\varepsilon}} \rightarrow 0$ as $\Lambda>R$.

Proof. From $W_{x, y} \geqq 0$ we get

$$
Z_{\Lambda}(\mathbf{q}) \leqq Z_{\Lambda}^{f}
$$

By the Jensen inequality we get:

$$
Z_{\Lambda}(\mathbf{q}) \geqq Z_{\Lambda}^{f} \exp -\sum_{x \in \Lambda} \sum_{y \in \Lambda}\left\langle W_{x, y}\left(\sigma_{x} \cdot-q_{y}\right)\right\rangle_{\Lambda}
$$


with an obvious definition for $\langle\cdot\rangle_{\Lambda}$.

From $A$, we can estimate the right hand side as follows

$$
\left\langle W_{x, y}\left(\sigma_{x}-q_{y}\right)\right\rangle_{\Lambda} \leqq J(x, y)\left\langle\left(1+\left\|\sigma_{x}\right\|\right)^{n}\right\rangle_{\Lambda}\left(1+\left|q_{y}\right|\right)^{n} .
$$

Now we claim that there is a constant $K_{3}$ such (see Lemma VI.3 below)

$$
\left\langle\left(1+\left|\sigma_{x}\right|\right)^{n}\right\rangle_{\Lambda} \leqq K_{3} \quad \forall x \in \Lambda \quad \forall \Lambda \in \mathfrak{F}(R) .
$$

Assuming this estimate we get

$$
\int d \mu \sum_{x \in \Lambda} \sum_{y \notin \Lambda}\left\langle W_{x, y}\left(\sigma_{x}-q_{y}\right)\right\rangle_{\Lambda} \leqq K_{4} \sum_{x \in \Lambda} \sum_{y \notin \Lambda} J(x, y)
$$

because, for $\mu \in \mathscr{G}_{t}(\mathscr{E}), \mu$ satisfies the "exponential" bound III.3, thus $\mu\left(\left(1+\left|\sigma_{y}\right|^{n}\right)\right.$ is uniformly bounded in $y \in R$.

Now let

$$
\Lambda_{1}=\left\{y \notin \Lambda ; \operatorname{dist}(\Lambda, y) \leqq|\Lambda|^{\varepsilon}\right\} .
$$

(Here $\partial \Lambda=\{y \notin \Lambda ; d(\Lambda, y) \leqq 1\}$.) Then

$$
\sum_{x \in \Lambda} \sum_{y \in \Lambda_{1}} J(x, y) \leqq\left\|J^{t}\right\||\partial \Lambda \| \Lambda|^{\varepsilon}
$$

if $J^{t}(x, y)=J(y, x)$. On the other hand,

$$
\sum_{x \in \Lambda} \sum_{y \notin \Lambda \cup \Lambda_{1}} J(x, y) \leqq|\Lambda|^{-\varepsilon N} \sum_{x \in \Lambda} \sum_{y \notin \Lambda \cup \Lambda_{1}} d(x, y)^{N} J(x, y) \leqq \frac{\|\mathbf{J}\|_{N}}{|\Lambda|^{\mid \varepsilon-1}} .
$$

Thus

$$
-\frac{1}{|\Lambda|} \log Z_{\Lambda}^{f} \leqq \int d \mu(\mathbf{q}) p_{\Lambda}(\mathbf{q}) \leqq-\frac{1}{|\Lambda|} \log Z_{\Lambda}^{f}+0\left(\frac{\partial \Lambda}{|\Lambda|^{1-\varepsilon}}\right)+0\left(\frac{1}{|\Lambda|^{\mid \varepsilon N}}\right)
$$

and the result follows.

It remains to prove:

Lemma VI.3. The estimate VI.15 holds.

To prove VI.15 it is sufficient to prove

$$
\left\langle\exp \left(a\left|\sigma_{x}\right|\right)\right\rangle_{\Lambda} \leqq \exp \left(\frac{A^{\prime} a^{2}}{2}+B^{\prime} a+C^{\prime}\right) \forall \Lambda \in \mathfrak{F}(R) a>0 .
$$

This estimate follows from III.1, where we have put $W_{x, y}=0$ unless $x \in \Lambda, y \in \Lambda$. Then, the matrix $r(x, y)$ does not depend on $\Lambda$.

VI.b) Uniqueness Criteria

Proposition VI.4. Let $\mathscr{E}$ be a compact, attractive local specification. Then $\mathscr{G}_{t}(\mathscr{E})$ has one point only, if and only if

$$
\mu_{+}\left(\sigma_{x}\right)=\mu_{-}\left(\sigma_{x}\right) \quad \forall x \in R
$$

Remark. Using the Lebowitz inequalities such a result can be proved in example [32]. However here we follow a rather general argument. 
Proof. By Proposition V.1 it is enough to prove that VI.22 implies $\mu_{+}=\mu_{-}$. Let $\mathbf{f}=\left(f_{x}\right)_{x \in R}$ be a positive rapidly decreasing sequence on $R$ and

$$
\sigma(\mathbf{f})=\sum_{x \in R} \sigma_{x} f_{x}
$$

Let $R_{\mathbf{f}}$ be the corresponding Wasershtein distance (see the Appendix). We get:

$$
R_{\mathbf{f}}\left(\mu_{+}, \mu_{-}\right)=\int_{-\infty}^{+\infty} d s\left[\mu_{+}\left(\chi_{[s, \infty]}(\sigma(\mathbf{f}))\right)-\mu_{-}\left(\chi_{[s, \infty]}(\sigma(\mathbf{f}))\right)\right] .
$$

Since $\mu_{+} \succ \mu_{-}$and $\chi_{[s, \infty]}(\sigma(\mathbf{f})) \in \mathscr{K}_{+}\left(\Omega_{R}\right)$, we can forget the absolute value to get

$$
R_{\mathbf{f}}\left(\mu_{+}, \mu_{-}\right)=\mu_{+}(\sigma(\mathbf{f}))-\mu_{-}(\sigma(\mathbf{f}))=\sum_{x \in R} f_{x}\left(\mu_{+}\left(\sigma_{x}\right)-\mu_{-}\left(\sigma_{x}\right)\right)=0,
$$

f being arbitrary, $\mu_{+}=\mu_{-}$.

Now let $h$ be a real number, and let $p(h)$ be the pressure corresponding to the model I.5 with $V$ replaced by

$$
V_{h}(q)=V(q)-h q .
$$

Clearly $p(h)$ is a concave function of $h$. We assume moreover that $W_{(x, y)}$ depends only on $x-y$, on $R=\mathbb{Z}^{v}$ (translation invariance). Then we get:

Proposition VI.5. Let us consider the model I.5, $A, B$, on $R=\mathbb{Z}^{v}$, with $W_{(x, y)}$ depending only on $(x-y) \in \mathbb{Z}^{v}$. Then if $p(h)$ is differentiable at $h=h_{0}$, the set of tempered Gibbs states corresponding to $V_{h_{0}}$ is reduced to one point.

Proof.

$$
\begin{gathered}
p(h)=\lim _{\Lambda \rtimes R} p_{\Lambda}^{\mu}(h), \\
p_{\Lambda}^{\mu}(h)=\int-\frac{1}{|\Lambda|} \log Z_{\Lambda}^{h}(\mathbf{q}) d \mu(\mathbf{q}) .
\end{gathered}
$$

Thus, $p_{A}^{\mu}(h)$ is concave and of class $\mathscr{C}^{1}$ in $h$. A well-known result on the concave functions $[17,24]$ allows us to give:

$$
\frac{\partial p\left(h_{0}\right)}{\partial h}=\lim _{\Lambda} \frac{\partial p_{\Lambda}^{\mu}\left(h_{0}\right)}{\partial h} .
$$

But

$$
\frac{\partial p_{\Lambda}^{\mu}\left(h_{0}\right)}{\partial h}=-\frac{1}{|\Lambda|} \int d \mu(\mathbf{q}) E_{\Lambda^{c}}^{\mathbf{q}}\left(\sum_{x \in \Lambda} \sigma_{x}\right)=-\frac{1}{|\Lambda|} \sum_{x \in \Lambda} \mu\left(\sigma_{x}\right) .
$$

In particular for $\mu=\mu_{ \pm}, \mu_{ \pm}\left(\sigma_{x}\right)=m_{ \pm} \forall x$, for $\mu_{ \pm}$are translation invariant (Prop V.3. Example 1). Thus

$$
\frac{\partial p\left(h_{0}\right)}{\partial h}=m_{+}=m_{-}
$$

since $\mu$ can be choosen arbitrarily in $\mathscr{G}_{t}(\mathscr{E})$. 
Using Proposition VI.4 the result is proved.

Example. Let us consider the $\varphi^{4}$ theory on the lattice:

$$
\left.\begin{array}{rl}
W_{x y}(q) & =\frac{1}{2} q^{2} \quad d(x, y)=1 \\
& =0 \text { otherwise } \\
V(q) & =\frac{1}{2}\left(m^{2}-\sigma^{2}\right) q^{2}+\lambda q^{4}, \quad \lambda>0 .
\end{array}\right\}
$$

The Lee-Yang theorem allows us to prove that $p(h)$ is analytic in $h$ for $\operatorname{Re}(h) \neq 0$ $[26,27,49]$. In this region we have the uniqueness of the Gibbs state:

Corollary VI.6. For the $\left(\lambda \varphi^{4}+b \varphi^{2}+h \varphi\right)$ theory on the lattice, the set of tempered Gibbs states is reduced to one point provided $h \neq 0$.

Remark. In this example, let $\mu^{h}$ be the unique Gibbs state corresponding to the case $h>0$. Then if $\mu$ is a Gibbs state for $h=0$, the FKG inequalities give:

$$
\mu \propto \mu^{h} .
$$

Thus, due to the compactness of $\mathscr{E}$

$$
\lim _{h \times O A \times R} \lim ^{h} E_{A^{c}}=\mu_{+}
$$

If $\mathscr{E}$ is the local specification corresponding to $h=0$. This construction was precisely done by Fröhlich and Simon [21] in the context of field theories to get the " + "state for the $P(\varphi)_{2}$ theory.

\section{Appendix: Tempered Measures}

As before $\Omega_{R}$ is the space $\mathbb{R}^{R}$ with the product topology; $\mathscr{S}_{R}^{\prime}$ is the subset of slowly increasing sequences in $\Omega_{R} . \mathscr{S}_{R}^{\prime}$ is equipped with the topology $\sigma\left(\mathscr{S}_{R}^{\prime}, \mathscr{S}_{R}\right)$, where $\mathscr{S}_{R}$ is the set of fastly decreasing sequences, by the canonical duality:

$$
\mathbf{q} \in \mathscr{S}_{R}^{\prime}, \mathbf{f} \in \mathscr{S}_{R}\langle\mathbf{q} \mid \mathbf{f}\rangle=\sum_{x \in R} q_{x} f_{x} .
$$

Since $\mathscr{S}_{R}$ is separable, if $\Sigma$ is a dense denumerable subspace of $\mathscr{S}_{R}$, the map

$$
\mathbf{q} \in \mathscr{S}_{R}^{\prime} \rightarrow(\langle\mathbf{q} \mid \mathbf{f}\rangle)_{\mathbf{f} \in \Sigma} \in \mathbb{R}^{\Sigma}=\Omega_{\Sigma}
$$

gives an homeomorphism between $\mathscr{S}_{R}^{\prime}$ and its image $\Sigma_{\mathscr{T}}$ in the topological space $\Omega_{\Sigma}$. It is well-known [48] that the weak topology and the strong topology on $\mathscr{S}_{R}^{\prime}$ coincide, that $\mathscr{S}_{R}^{\prime}$ is a polish space, and that a subset $B$ of $\mathscr{S}_{R}^{\prime}$ is compact if and only if there are $C_{B}>0$ and $N_{B} \in N$ such that

$$
\mathbf{q} \in B \Rightarrow\left|q_{x}\right| \leqq C_{B}(1+d(0, x))^{N_{B}}
$$

Now let $\mathfrak{M}_{t}\left(\Omega_{R}\right)$ be the set of tempered measures on $\Omega_{R}$. For $\mathbf{f}$ belonging to $\mathscr{S}_{R}^{+}=\left\{\mathbf{f} \in \mathscr{S}_{R} ; f_{x} \geqq 0 \forall x\right\}$ we put

$$
\rho_{\mathbf{f}}(\mathbf{q})=\sum_{x \in R} f_{x}\left|q_{x}\right| .
$$


Then $\mu \in \mathfrak{M}_{t}\left(\Omega_{R}\right)$ if and only if $\mu \in \mathfrak{M}\left(\Omega_{R}\right)$ and

$$
\mu\left(\rho_{\mathbf{f}}\right)<+\infty \quad \forall \mathbf{f} \in \Sigma .
$$

Let $\mathscr{W}$ be the weak topology on $\mathfrak{M}$, and we equip $\mathfrak{M}_{t}$ with the induced topology.

Proposition A.1. [39] For every $\mu \in \mathfrak{M}_{t}$, the subset $\mathscr{S}_{R}^{\prime}$ of $\Omega_{R}$ has $\mu$-measure one. Proof. We get easily, using the Tchebischef inequality

$$
\begin{aligned}
\mu\left(\Omega_{R} \backslash \mathscr{S}_{R}^{\prime}\right) & \leqq \mu\left\{\mathbf{q} \in \Omega_{R} ; \exists x \in R\left|q_{x}\right| \geqq \frac{1}{\varepsilon}(1+d(0, x))^{N}\right\} \\
& \leqq \varepsilon \sum_{x \in R} \frac{\mu\left(\left|\sigma_{x}\right|\right)}{(1+d(0, x))^{N} .}
\end{aligned}
$$

Choosing $N$ big enough and $\varepsilon$ as small as we want, we get the result.

Proposition A.2. [39] Let $C$ be a positive real number and $N$ be an integer. The set

$$
\mathfrak{E}(C, N)=\left\{\mu \in \mathfrak{M}_{t}\left(\Omega_{R}\right) ; \mu\left(\left|\sigma_{x}\right|\right) \leqq C(1+d(0, x))^{N}\right\}
$$

is compact in $\left(\mathfrak{M}_{t}, \mathscr{W}\right)$ and

$$
\mathfrak{M}_{t}=\bigcup_{C \in N, N \in \mathbb{N}} \mathfrak{E}(C, N)
$$

Proof. Giving $\varepsilon>0, C>0$ and $N \in \mathbb{N}$, we define

$$
\mathscr{K}_{\varepsilon}=\left\{\mathbf{q} \in \Omega_{R} ;\left|q_{x}\right| \leqq \frac{C}{\varepsilon}(1+d(0, x))^{N+v+1}\right\} .
$$

By the Tychonov theorem $\mathscr{K}_{\varepsilon}$ is compact in $\Omega_{R}$, and

$$
\mu\left(\Omega_{R} \backslash \mathscr{K}_{\varepsilon}\right) \leqq \varepsilon \sum_{x \in R} \frac{\mu\left(\left|\sigma_{x}\right|\right)}{C(1+d(0, x))^{N+v+1}} \leqq \varepsilon \sum_{x \in R} \frac{1}{(1+d(0, x))^{N+v+1}}
$$

if $\mu \in \mathfrak{E}(C, N)$.

By the Prokhorov theorem [42], $\mathfrak{E}(C, N)$ has a compact closure. But $\mu \stackrel{\Phi}{\rightarrow}\left(\mu\left(\left|\sigma_{x}\right|\right)\right)_{x \in R}$ is lower semi-continuous, and therefore $\mathfrak{E}(C, N)$ is closed as an inverse image of a closed set by $\Phi$.

Corollary A.3. $\left(\mathfrak{M}_{t}, \mathscr{W}\right)$ is a Suslin space [8].

Proof. $\mathfrak{M}_{t}$ is the denumerable union of compact metrizable space. [see [8], Sect.6 n.2, Prop 8]

Let $\mathfrak{M}_{1}(\mathbb{R})$ be the set of probability measures on the real line such that

$$
\int_{-\infty}^{+\infty} d \mu(s)|s|<+\infty
$$

On $\mathfrak{M}_{1}(\mathbb{R})$ the Vasershtein distance can be defined as follows $[6,14,16,50]$

$$
R(\mu, v)=\int_{-\infty}^{+\infty} d s\left|\mu\left(\chi_{[s, \infty]}\right)-v\left(\chi_{[s, \infty]}\right)\right|
$$


where $\chi_{[s, \infty]}$ is the characteristic function of $[s, \infty]$. It is known that $\left(\mathfrak{M}_{1}(\mathbb{R}), R\right)$ is a complete metric space with a stronger topology than the weak topology. Giving $\mathbf{f} \in \mathscr{S}_{R}$ we define

$$
\sigma_{\mathbf{f}}=\sum_{x \in R} \sigma_{x} f_{x}
$$

and for $\mu \in \mathfrak{M}_{t}\left(\Omega_{R}\right)$ let $\mu_{\mathrm{f}}$ be the measure on the $\mathbb{R}$ image of $\mu$ by $\sigma_{\mathbf{f}}$. Since $\mu \in \mathfrak{M}_{t}$, $\mu_{\mathbf{f}} \in \mathfrak{M}_{1}(\mathbb{R})$. Then we define

$$
R_{\mathbf{f}}(\mu, v)=R\left(\mu_{\mathbf{f}}, v_{\mathbf{f}}\right) \quad \mathbf{f} \in \mathscr{S}_{R}
$$

Proposition A.4. [39] The family $\left(R_{\mathbf{f}}\right)_{\mathbf{f} \in \mathscr{S}_{R}}$ defines a topology $\mathscr{T}$ on $\mathfrak{M}_{t}$, for which $\left(\mathfrak{M}_{t}, \mathscr{T}\right)$ is a polish space. $\mathscr{T}$ is stronger than $\mathscr{W}$.

Proof. We first note that $\mathbf{f} \in \mathscr{S}_{R} \rightarrow R_{\mathrm{f}}$ is continuous because

$$
\left|R_{\mathbf{f}}(\mu, v)-R_{\mathbf{g}}(\mu, v)\right| \leqq R_{\mathbf{f}-\mathbf{g}}(\mu, v) .
$$

For $R_{\mathbf{f}}$ can be defined as:

$$
R_{\mathbf{f}}(\mu, v)=\inf \left\{\int d m(\mathbf{p}, \mathbf{q})\left|\sigma_{\mathbf{f}}(\mathbf{p})-\sigma_{\mathbf{f}}(\mathbf{q})\right| ; m \in W(\mu, v)\right\},
$$

where $W(\mu, v)$ is the set of probability measures on $\Omega_{R} \times \Omega_{R}$, the projections of which are $\mu$ and $v$ respectively.

On the other hand

$$
R_{\mathbf{f}}(\mu, v) \leqq \mu\left(\left|\sigma_{\mathbf{f}}\right|\right)+v\left(\left|\sigma_{\mathbf{f}}\right|\right) \leqq \sum_{x \in R}\left|f_{x}\right|\left(\mu\left(\left|\sigma_{x}\right|\right)+v\left(\left|\sigma_{x}\right|\right)\right) .
$$

It follows that if $\Sigma$ is denumerable and dense in $\mathscr{S}_{R}$, the family $\left(R_{\mathrm{f}}\right)_{\mathbf{f} \in \Sigma}$ defines the same topology $\mathscr{T}$. Thus $\mathscr{T}$ is metrizable and complete because (see A.16)

$$
\left|\mu\left(\left|\sigma_{\mathbf{f}}\right|\right)-v\left(\left|\sigma_{\mathbf{f}}\right|\right)\right| \leqq R_{\mathbf{f}}(\mu, v) .
$$

Moreover if $\mu \in \mathfrak{M}\left(\Omega_{R}\right)$ and $p_{\mu}(\mathbf{f})=\mu\left(\left|\sigma_{\mathbf{f}}\right|\right)<+\infty \forall \mathbf{f} \in \mathscr{S}_{R}$, then $p_{\mu}$ is a continuous semi-norm and therefore $\mu \in \mathfrak{M}_{t}$.

At last $\mathscr{T}$ is Hausdorff because $R_{\mathbf{f}}(\mu, v)=0 \forall \mathbf{f}$ implies $\mu_{\mathbf{f}}=v_{\mathbf{f}} \forall \mathbf{f} \in \mathscr{S}_{R}$, which, by the Kolmogorov theorem is equivalent to $\mu=v$.

The fact that $\mathscr{T}$ is stronger than $\mathscr{W}$ is a consequence of the definition of $R_{\mathrm{f}}$ $[[14]$, theorem 2].

Definition A.5. A subset $H$ of $\mathfrak{M}_{t}$ is called uniform if for any $\mathbf{f} \in \mathscr{S}_{\boldsymbol{R}}$

$$
\lim _{A \rightarrow \infty} \int_{|\sigma| \mathbf{f} \geqq A} d \mu\left|\sigma_{\mathbf{f}}\right|=0
$$

uniformly for $\mu \in H$.

The great interest of this definition comes from the following characterization of compact subset of $\left(\mathfrak{M}_{t}, \mathscr{T}\right)$.

Proposition A.6. A subset $H$ of $\left(\mathfrak{M}_{t}, \mathscr{T}\right)$, has a compact closure if and only if the following conditions hold.

(i) $H$ is uniform.

(ii) $\exists C>0, N \in \mathbb{N}, \quad H \subset \mathbb{E}(C, N)$. 
Proof. Let us assume $H$ to be $\mathscr{T}$-compact.

Since $\forall \mathbf{f} \in \mathscr{S}_{R} \mu \rightarrow \mu\left(\left|\sigma_{\mathbf{f}}\right|\right)$ is continuous, for the $\mathscr{T}$ topology the semi-norm

$$
p_{H}(\mathbf{f})=\sup _{\mu \in \boldsymbol{H}} \mu\left(\left|\sigma_{\mathbf{f}}\right|\right)
$$

is everywhere defined and lower semi-continuous on $\mathscr{S}_{R}$. Since $\mathscr{S}_{R}^{\prime}$ is a Baire space, $p_{H}$ is continuous, $([8] \S 5)$ and we can find $C>0, N \in \mathbb{N}$ such that

$$
p_{H}(\mathbf{f}) \leqq C \sum_{x \in R}|1+d(x, 0)|^{N}\left|f_{x}\right|
$$

Thus $H \subset \mathfrak{E}(C, N)$.

Moreover let $\varepsilon$ be positive, and $\mathbf{f} \in \mathscr{S}_{R}$; then there are $\mu_{1}, \ldots, \mu_{n}, \mu \in H$, such that $\forall \mu \in H$

$$
\inf _{j} R_{\mathbf{f}}\left(\mu, \mu_{j}\right) \leqq \varepsilon / 4
$$

Let $F_{A}$ be the function on $\mathbb{R}$,

$$
\begin{aligned}
F_{A} & =0|p| \leqq A / 2 ; F_{A}(p)=2|p|-A, A / 2 \leqq|p| \leqq A \\
F_{A}(p) & =|p|,|p| \geqq A .
\end{aligned}
$$

Then: $\left|F_{A}(p)-F_{A}(q)\right| \leqq 2|p-q|$ and

$$
\int_{|\sigma \mathbf{f}| \geqq A} d \mu\left|\sigma_{\mathbf{f}}\right| \leqq \int d \mu F_{A}\left(\sigma_{\mathbf{f}}\right) \leqq 2 R_{\mathbf{f}}\left(\mu, \mu_{j}\right)+\int d \mu_{j} F_{A}\left(\sigma_{\mathbf{f}}\right) .
$$

Choosing $A(\varepsilon ; \mathbf{f})$ such that $A \geqq A(\varepsilon, \mathbf{f})$ implies

$$
\int d \mu_{j} F_{A}\left(\sigma_{\mathbf{f}}\right) \leqq \varepsilon / 2, \quad j=1, \ldots, n
$$

we get

$$
\int_{\left|\sigma_{\mathbf{f}}\right| \geqq A} d \mu\left|\sigma_{\mathbf{f}}\right| \leqq \varepsilon, \quad \mu \in H
$$

and $H$ is a uniform set.

Conversely if $H$ satisfies (i) and (ii), $H$ is weakly relatively compact. But since $H$ is uniform $\mathscr{T}$ and $\mathscr{W}$ coincide on $H$ by the result of Dobrushin [14, Theorem 2]. Then, $H$ is $\mathscr{T}$ relatively compact.

Remark A.7. We note now that in Sect.III, the exponential bound is sufficient to insure that $\mathscr{E}$ is compact in the sense of the $\mathscr{T}$-topology. For if $\chi_{A_{0}}(p)=1$ if $p \geqq A_{0}=$ 0 otherwise

$$
\exists C(a)>0,\left|\sigma_{\mathbf{f}}\right| \chi_{A_{0}}\left(\sigma_{\mathbf{f}}\right) \leqq A_{0} \exp \left(a\left(\sigma_{\mathbf{f}}-A_{0}\right)\right) C(a)
$$

and

$$
\mu\left(\left|\sigma_{\mathbf{f}}\right| \chi_{A_{0}}\left(\sigma_{\mathbf{f}}\right)\right) \leqq A_{0} \exp \left(-A_{0} a\right) C(a) \mu\left(\exp \left(a \sigma_{\mathbf{f}}\right)\right), \quad \mu \in \mathfrak{M}
$$

This estimate proves that if $\rho \in \mathfrak{M}_{t}$

$$
\lim _{A \rightarrow \infty} \rho E_{A^{c}}\left(\chi_{A}\left(\sigma_{\mathbf{f}}\right)\left|\sigma_{\mathbf{f}}\right|\right)=0
$$


uniformly in $\Lambda$, and

$$
\lim _{A \rightarrow \infty} \mu\left(\chi_{A}\left(\sigma_{\mathbf{f}}\right)\left|\sigma_{\mathbf{f}}\right|\right)=0
$$

uniformly in $\mu \in \mathscr{G}_{t}(\mathscr{E})$, because $\mathscr{E}$ satisfies the exponential bound.

Acknowledgements. This paper was written partly in Marseille and partly in Oslo, and it is a pleasure to thank Matematisk Institutt, Universitetet i Oslo as Faculté de Sciences Luminy, Université d'Aix, Marseille, for the assistance which made this work possible. In addition it was also possible for the authors to meet at the Fakultät für Physik, Bielefeld Universität, and for this we are also grateful. We also want to thank Professors R.L. Dobrushin, S. Albeverio, C. Preston and E. Presutti for constructive and instructive discussions through various places in the completion of this paper. We also want to thank Mrs. Sigrid Cordtsen for expert typing through a not always very clear manuscript.

\section{References}

1. Albeverio, S., and Hogh-Krohn, R. : Topics in infinite dimensional analysis. In: Mathematical problems in theoretical physics. Dell' Antonio, G. F., Doplicher, S. Jona-Lasinio, G. (eds.) Lecture Notes in Physics, Vol. 80, Berlin-Heidelberg-New York : Springer 1970

2. Albeverio, S., Hфegh-Krohn, R. : Local and global Markov field. Karpacz Winter School (1978)

3. Albeverio, S., H $\phi$ egh-Krohn, R. : The Markoff property for Euclidean fields. The case of trigonometric interaction. Commun. Math. Phys. 68, 95-128(1979)

4. Albeverio, S., Hфegh-Krohn; R., Olsen, G. : The global Markov property for lattice systems. Oslo Preprint (1978) See Phys. Lett. 84B, 89-90 (1979)

5. Benfatto, G., Presutti, E., Pulvirenti, M. : DLR measure for one dimensional harmonic systems. Z. Wahrscheinlich-tsch keitsth. Verw. Geb. 41, 305-312 (1978)

6. Benfatto, G., Cassandro, M., Kipnis, C., Olivieri, E., Pelle-grinotti, A., Presutti, E. : An explicit formula for the Vasershtein distance for probability measure on $\mathbb{R}$. Publicazione dell'Istituto di Matematica, Universita dell'Aquila (1977)

7. Cassandro, M., Olivieri, E., Pellegrinotti, A., Presutti, E. : Existence and ūniqueness of DLR measures for unbounded spin system. Z. Wahrscheinlichkeitsth. Verw. Geb. 41, 313-334 (1978)

8. Bourbaki, N. : Topologie général. Chap. IX. Paris: Hermann, 1958

9a. Choquet, G. : Lectures on analysis, Vol. II. Marsden, J., Lance, T., Gelbart, S. (eds). New York, Amsterdam: Benjamin Inc, 1969

b. Bourbaki, N. : Integration Chap. IV, Sect. 7, Paris: Hermann 1965

10. Dobrushin, R. L. : Gibbsian random fields for lattice systems with pairwise interactions. Funct. Anal. Appl. 2, 292-301 (1968)

11. Dobrushin, R. L. : The problem of ūniqueness of a Gibbsian random field and the problem of phase transition. Funct. Anal. Appl. 2, 302-312 (1968).

12. Dobrushin, R. L. : Gibbsian random fields. General case. Funct. Anal. Appl. 3, 22 (1969).

13. Dobrushin, R. L. : The description of a random field by means of conditional probabilities and conditions of its regularity. Theor. Prob. Appl. 13, 197-224 (1968)

14. Dobrushin, R. L. : Prescribing a system of random variables by conditional distribution. Theor. Prob. Appl. 13, 458-486 (1970)

15. Dobrushin, R. L., Minlos, R. A. : The theory of Markov random fields and its application to quantum field theory. In: Functional and probabilistic methods in quantum field theory. Vol. 1. p. 23-49, Jancewicz, (ed.) Acta Univ. Wratislava No 369 Wroclaw 1976

16. Dobrushin, R. L., Pecherskyi : In: Proceeding of the Colloquium on random fields: Rigorous results in statistical mechanics and quantum field theory. Esztergom, Hungary 1979

17. Fischer, M. E. : Correlation functions and coexistance of phases. J. Math. Phys. 6, 1643-1653 (1965) Lemma III

18. Fölmer, H. : On the potential theory of stochastic fields. Invited address 40th Session ISI, Warsaw, Poland (1975) 
19. Fölmer, H. : On the global Markov property. In: Quantum fields-algebras-processes. Streit, L. (ed.) Wien, New York: Springer, p. 293-302, 1980

20. Fortuin, C., Kasteleyn, P., Ginibre, J. : Correlation inequalities on some partially oriented sets. Commun. Math. Phys. 22, 89-103 (1971)

21. Fröhlich, J., Simon, B. : Pure states for general $\mathrm{P}(\varphi)_{2}$ theories: Construction, regularity, variational equalities. Ann. Math. 105, 493-526 (1977)

22. Glimm, J., Jaffe, A., Spencer, T. : Phase transition for $\varphi_{2}^{4}$ quantum fields. Commun. Math. Phys. 45, 203 (1975)

23. Glimm, J., Jaffe, A., Spencer, T. : Convergent expansion about mean field theory, Part I and II. Ann. Phys. 101, 610-630, 631-669 (1976)

24. Griffiths, R. B. : A proof that the free energy of spinny system is extensive. J. Math. Phys. 5, 1215-1222 (1964) Appendix A

25. Gross, L. : Decay of correlations in classical lattice models at high temperature. Commun Math. Phys. 68, 9 (1979)

26. Guerra, F., Rosen, L., Simon, B. : The $P(\varphi)_{2}$ quantum field theory as classical statistical mechanics. Ann. Math. 101, 111-259 (1975)

27. Guerra, F., Rosen, L., Simon, B. : In: Constructive quantum field theory. Lecture Notes in Physics Vol. 25. Velo, G., Wightman, A. S. (eds.) Berlin, Heidelberg, New York: Springer (1973)

28. Guerra, F., Rosen, L., Simon, B. : Boundary conditions for the $\mathrm{P}(\varphi)_{2}$ Euclidean field theory. Ann. Inst. H. Poincaré, Vol. 25, $231-334$ (1976)

29. Lanford, O. III: In: Statistical mechanics and mathematical problems. Lecture Notes in Physics 20, Ed. A. Lenard, Battelle Rencontres, Seattle 1971, p. 1-107, Berlin: Springer-Verlag, 1973.

30. Lanford, O. III, Ruelle, D. : Observables at infinity. Commun. Math. Phys. 13, 194 (1969)

31. Lebowitz, J. L., Presutti, E. : Statistical mechanics of unbounded spins systems. Commun. Math. Phys. 50, 195-218 (1976)

32. Lebowitz, J. L. : GHS and other inequalities. Commun. Math. Phys. 35, 87-92 (1974)

33. Nelson, E. : Quantum fields and Markov fields. In : Partial differential equations. Spencer, D. (ed.) Symp. Pure Math. AMS Vol. 23, 413-420 (1973)

34. Nelson, E. : Construction of quantum fields from Markov fields. J. Funct. Anal. 12, 97-112 (1973)

35. Nelson, E. : The free Markoff field. J. Funct. Anal. 12, 211-223 (1973)

36. Nelson, E. : Probability theory and Euclidean field theory. In: Constructive quantum field theory. Velo, G., Wightman, A. S. (eds.) Lecture Notes in Physics Vol. 25, Berlin, Heidelberg, New York: Springer 1975

37. Newman, C. : The construction of stationary two dimensional Markoff fields with an application to quantum field theory. J. Funct. Anal. 12, 221-277 (1973)

38. Parthasarathy, K. R. : Probability measures on metric spaces. New York: Academic Press 1968

39. Picco, P. : These de $3^{e}$ cycle. Marseille (1979)

40. Preston, C. : Random fields. In: Lecture Notes in Mathematics, Vol. 534, Berlin: Springer 1975, and references therein

41. Preston, C. : Construction of specifications. In: Quantum fields, algebras, processes. Streit, L. (ed.) pp. 269-292. Wien, New York: Springer 1980

42. Prokhoroff, L. : Convergence of random processes and limit theorem in probability theory. Theor. Prob. Appl. 1, 157-214 (1956)

43. a) Rosen, L. : Correlation inequalities for $Y_{2}$ quantum field theory, Proceedings of the Colloquium on random fields: Rigorous results in statistical mechanics and quantum field theory (Esztergom, Hungary, 1979)

b) Battle, G. A., Rosen, L. : The FKG inequality for the Yukawa ${ }_{2}$ quantum field theory. J. Stat. Phys. 22, 123-192 (1980) Theorem 1.1.

44. Ruelle, D. : Statistical mechanics. Rigorous results, New York: Benjamin 1969

45. Ruelle, D. : Thermodynamical formalism. Reading, Mass. : Addison Wesley Pub. Co. 1978 
46. Ruelle, D.: Superstable interaction in classical statistical mechanics. Commun. Math. Phys. 18, 127 (1970)

47. Ruelle, D. : Probability estimates for continuous spin system. Commun. Math. Phys. 50, 189-194 (1976)

48. Schwartz, L. : Theorie des distribution. Paris: Hermann 1966

49. Simon, B.: The $\mathrm{P}(\varphi)_{2}$ Euclidean quantum field theory. Princeton Series in Physics, Princeton, N. J. : Princeton University Press 1974

50. Vasershtein, L. N. : Markov processes over a countable product space describing large systems of automata. Prob. in Trans of Information, Vol. 5, 64-72 (1969) (English translation)

51. Loêve, M. : Probability theory. (3rd edn.) Princeton, New Jersey, New York, Toronto, London: Van Nostrand Comp. 1963. (See Sect. 21)

52. Albeverio, S., Fenstad, J. E., Lindstr $\phi$ m, T., H $\phi$ egh-Krohn, R.: Non-standard methods in mathematical physics and stochastic analysis. (in preparation)

Communicated by E. Lieb

Received May 20, 1981 
